\title{
CAA of an Air-Cooling System for Electronic Devices
}

\author{
Sven Münsterjohann, ${ }^{1}$ Jens Grabinger, ${ }^{2}$ Stefan Becker, ${ }^{1}$ and Manfred Kaltenbacher ${ }^{3}$ \\ ${ }^{1}$ Friedrich-Alexander-Universität Erlangen-Nürnberg, Cauerstr. 4, 91058 Erlangen, Germany \\ ${ }^{2}$ SIMetris GmbH, Am Weichselgarten 7, 91058 Erlangen, Germany \\ ${ }^{3}$ Vienna University of Technology, Getreidemarkt 9, 1060 Wien, Austria \\ Correspondence should be addressed to Sven Münsterjohann; sven.muensterjohann@fau.de
}

Received 4 July 2016; Revised 31 August 2016; Accepted 7 September 2016

Academic Editor: Marc Thomas

Copyright (C) 2016 Sven Münsterjohann et al. This is an open access article distributed under the Creative Commons Attribution License, which permits unrestricted use, distribution, and reproduction in any medium, provided the original work is properly cited.

\begin{abstract}
This paper presents the workflow and the results of fluid dynamics and aeroacoustic simulations for an air-cooling system as used in electronic devices. The setup represents a generic electronic device with several electronic assemblies with forced convection cooling by two axial fans. The aeroacoustic performance is computed using a hybrid method. In a first step, two unsteady CFD simulations using the Unsteady Reynolds-Averaged Navier-Stokes simulation with Shear Stress Transport (URANS-SST) turbulence model and the Scale Adaptive Simulation with Shear Stress Transport (SAS-SST) models were performed. Based on the unsteady flow results, the acoustic source terms were calculated using Lighthill's acoustic analogy. Propagation of the flow-induced sound was computed using the Finite Element Method. Finally, the results of the acoustic simulation are compared with measurements and show good agreement.
\end{abstract}

\section{Introduction}

In today's world, the dimensions of electronic components are continuously decreasing. While many electronic devices are consuming less energy than a few years ago, the density of the chips assembled on a printed circuit board is increasing. This higher density leads to less heat dissipation by natural convection, which demands forced convection in order to prevent overheating. Convection can be forced by water or air cooling, with the latter using radial or axial fans to generate an air flow through the devices. As many of these air-cooled devices, measurement equipment or video game consoles, are operated by humans, their noise emission is an important attribute. On the one hand, the noise emission has to be low to please the users; on the other hand, these devices have to meet strict industrial regulations with a maximum allowable noise emission in the working environment. To meet these requirements, the prediction of the noise emission is an essential tool to avoid claims and restrictions. In addition to the experimental investigation of prototypes, the numerical estimation of the noise emission is a useful tool even before a first prototype is built. Acoustic measurements mostly provide an overall sound pressure level of the device without the possibility of closer detection of the acoustic sources. An exception is the microphone array measurement, but the spatial resolution depends strongly on the frequency under investigation. Furthermore, nearly all electronic devices have housing that makes the localization of acoustic sources on the inside virtually impossible. Numerical simulations are not subject to these restrictions and thus provide a strong tool for acoustic optimizations.

In this paper, the numerical calculation of the noise emission of a generic electronic 19-inch slide-in device with two $120 \mathrm{~mm}$ axial fans is presented. The computation facilitated an aeroacoustic hybrid approach that uses the Finite Volume (FV) solver ANSYS CFX for the flow simulation and the inhouse Finite Element (FE) solver CFS++ [1] for the acoustic simulation. The whole process starting from generation of the CAD model, CFD mesh generation, CFD setup, and solving to acoustic mesh generation and simulation of acoustic wave propagation is described. Two different turbulence models, URANS-SST and SAS-SST, will be compared with a focus on flow field quantities, that is, pressure and velocity, and sound pressure. While the URANS-SST model is only capable of resolving large scaled, triggered phenomena like vortex shedding behind a cylinder or rotor-stator interactions, 
the SAS-SST model can resolve the turbulence, given that temporal and spatial discretization are fine enough. This will especially improve the acoustic results in frequency ranges where broadband noise caused by turbulence is generated that cannot be resolved by the URANS model. Hence, the SAS-SST model is a good choice for capturing systemtriggered as well as turbulence generated noise.

\section{Aeroacoustics in Cooling Systems}

Most work performed on the acoustics of cooling systems in electronic devices, that is, computers or notebooks, is done experimentally. Baugh [2] investigated the changes in hydrodynamic and acoustic behavior due to the inlet restrictions in notebooks. By scaling the hydrodynamic performance of fans to isoacoustic fan curves under the condition of inlet restrictions, Baugh found a way to compare the in-system performance and acoustic behavior of different fans if the pressure drop in the system is known. Nantais et al. [3] performed acoustic measurements on the cooling solutions for different graphics processing units and found that the noise to be emitted was dependent not only on the fan size and the cooling requirements, but also on the circumferential speed of the fan and the design of the cooling system. Huang's group carried out research on computer cooling fan noise. Huang [4] used analytic and empirical models to study the influence of rotor-stator interaction by decomposing this source into axial thrust, circumferential speed, and radial force using point force formulation. He investigated the influence of the number of blades and motor struts and the distribution of the source on the noise emission. Later, Huang and Wang [5] combined experimental investigations and an analysis of the rotor-stator interaction with the point source formulation. They found three main sources within the investigated fourstrutted seven-bladed fan: the interaction between the blades and the struts, the additional size of one strut holding the electric wiring for the motor, and the incomplete bell mouth at the intake of the fan.

So far, most work has been performed by applying experimental and/or analytic approaches. Defoe and Novak [6] gave a brief review of methods for computational aeroacoustic (CAA) in electronic devices. They discussed different CAA methods: direct CAA, acoustic analogies, boundary element methods, and broadband methods. Comparing the results obtained by other authors with these different methods, the conclusion was drawn that the use of acoustic analogy is the best trade-off between computational effort and a detailed description of the acoustic sound field.

Using numerical methods our intention is to provide further insight into the sound generation in air-cooling systems of electronic devices. With a combination of CFD and CAA and modal analysis of the housing, a wide range of effects in the final acoustic spectra can be explained.

\section{Theory}

Various approaches have been proposed for both flow simulation and acoustic simulation, and the theories behind these approaches are explained in Sections 3.1 and 3.2.
3.1. Flow Simulation. The turbulence models applied in the flow simulation are the Shear Stress Transport (SST) turbulence model and the Scale Adaptive Simulation (SAS) developed by Menter [7]. As the acoustic source terms are directly derived from the flow field, sufficient resolution of the flow phenomena producing the acoustic sound emission is essential. The unsteady flow field can be simulated using different turbulence modeling approaches. The Unsteady Reynolds-Averaged Navier-Stokes equations in combination with the $k-\epsilon$, the $k-\omega$, or the SST model are the common way with the least demands regarding spatial and temporal resolution. More information is gained by using the SAS model, which adapts the turbulence modeling with respect to the spatial and temporal resolution. Further details of the flow field are simulated by a detached eddy simulation (DES) or a large eddy simulation (LES). In contrast to the SAS model, which falls back to a URANS solution when the discretization (temporal or spatial) is too coarse, the DES or LES model has special requirements regarding discretization. The best, but also the most expensive, solution is retrieved by a direct numerical simulation (DNS) of the flow. In this case, the discretization must be able to resolve the majority of the turbulent scales in time and space.

The CFD simulations in this paper were performed using the URANS-SST model and the SAS-SST model.

3.1.1. Shear Stress Transport (SST) Turbulence Model. In the SST $k-\omega$ model Menter [7] combined the advantages of the $k-\omega$ model [8] and $k-\epsilon$ model [9]. Although the $k-\epsilon$ model has its benefits in free stream regions, the $k$ - $\omega$ model leads to a more physical resolution of the flow in nearwall regions. With that knowledge, Menter derived the SST $k-\omega$ model: two functions, $F_{1}$ and $F_{2}$, dynamically blend between the original $k-\omega$ model and a transformed $k-\epsilon$ model. The blending is dependent on the wall distance $y$ and the thickness of the boundary layer at that location. The twoequation set of the $k-\omega$ model is multiplied by blending function $F_{1}$. Accordingly, the transformed $k-\epsilon$ model, which has a transport equation for $\epsilon$ depending on $\omega$, is multiplied by $\left(1-F_{1}\right)$. The corresponding transport equations of the models are added, resulting in the equation set of the SST $k-\omega$ model [7].

3.1.2. Scale Adaptive Simulation (SAS) Approach. Three types of two-equation turbulence models have been developed in the past: the $k-\omega$ model by Kolmogorov [9], the $k-\epsilon$ model by Launder and Spalding [8], and the $k-k L$ model by Rotta [10]. Compared with the $k-\omega$ and $k-\epsilon$ transport equations, Rotta found a formulation of the transport equations that hold a natural length scale. The advantage of this length scale is the possibility of dynamically reacting to resolved structures within the flow. In turn, the first and third spatial derivatives of the velocity are inherent in the transport equations.

Based on Rotta's formulation, Menter and Egorov [11, 12] showed that turbulent transport equations include the second instead of the third derivative of the velocity (as used in Rotta's approach). The turbulent transport quantities form a $\sqrt{k} L$ model that is known as the SAS model (Scale 
Adaptive Simulation). The first formulation of the model [12] has undergone some modifications, resulting in the current version of the SAS model [13], which is also implemented in ANSYS CFX 14.0+. Assuming that the turbulent structure is resolved within the flow field and not modeled, the turbulent frequency $\omega$ is increased and finally less modeling of the turbulence occurs due to a reduced eddy viscosity. The advantage of the model is obvious: the SAS-SST model dynamically reduces the modeling provided that turbulent structures can be resolved within the flow field (except for a limiter function to keep a minimum value for eddy viscosity [14]).

3.2. Acoustic Simulation. The process used here to compute the flow-induced sound is a hybrid method; that is, the acoustic simulation is performed as a second step after the results of the flow simulation have become available. The propagation of flow-induced sound is governed by Lighthill's inhomogeneous wave equation [15]:

$$
\frac{1}{c_{0}^{2}} \frac{\partial^{2} p^{\prime}}{\partial t^{2}}-\frac{\partial^{2} p^{\prime}}{\partial x_{i}^{2}}=\frac{\partial^{2} \mathbf{T}_{i j}}{\partial x_{i} \partial x_{j}}
$$

where $c_{0}$ is the average speed of sound in air and $p^{\prime}$ is a fluctuating pressure, which approaches the acoustic pressure outside the flow region (for details, see [1]). The wave equation is loaded on the right-hand side with the secondorder spatial derivative of the Lighthill tensor:

$$
\mathbf{T}_{i j}=\rho u_{i} u_{j}+\left(\left(p-p_{0}\right)-c_{0}^{2}\left(\rho-\rho_{0}\right)\right) \delta_{i j}-\tau_{i j},
$$

which depends on data for the turbulent flow precomputed by the flow simulation, such as the velocity $u_{i}$, the aerodynamic pressure $p$, the density $\rho$, and the viscous stress tensor $\tau_{i j}$. For isentropic flow at low Mach number, this can be approximated by $\mathbf{T}_{i j} \approx \rho_{0} u_{i} u_{j}$, where $\rho_{0}$ is the average density of air.

The inhomogeneous wave equation is solved using the Finite Element Method (FEM). Before the spatial and temporal discretization can be applied, the variational formulation of (1) must be derived [16]. This is done by multiplying with a test function $w$ and integrating the equation over the simulation domain $\Omega$ :

$$
\int_{\Omega} w\left(\frac{1}{c_{0}^{2}} \frac{\partial^{2} p^{\prime}}{\partial t^{2}}-\frac{\partial^{2} p^{\prime}}{\partial x_{i}^{2}}-\frac{\partial^{2} \mathbf{T}_{i j}}{\partial x_{i} \partial x_{j}}\right) \mathrm{d} \Omega=0 .
$$

Green's integral theorem is then applied in order to reduce the second-order spatial derivatives to first order:

$$
\begin{aligned}
& \int_{\Omega} \frac{1}{c_{0}^{2}} w \frac{\partial^{2} p^{\prime}}{\partial t^{2}} \mathrm{~d} \Omega+\int_{\Omega} \frac{\partial w}{\partial x_{i}} \frac{\partial p^{\prime}}{\partial x_{i}} \mathrm{~d} \Omega-\int_{\Gamma} w \frac{\partial p^{\prime}}{\partial \mathbf{n}} \mathrm{d} \Gamma \\
&=-\int_{\Omega} \frac{\partial w}{\partial x_{i}} \frac{\partial \mathbf{T}_{i j}}{\partial x_{j}} \mathrm{~d} \Omega,
\end{aligned}
$$

where $\Gamma=\partial \Omega$ is the boundary of the simulation domain and $\mathbf{n}$ is the surface normal vector on $\Gamma$. If we set the boundary integral to zero, an acoustically hard wall (i.e., ideally reflecting) is assumed at the boundary. In order to achieve free-field radiation where the boundary is purely artificial (denoted by $\Gamma_{a}$ ), we use an absorbing boundary condition expressed by

$$
\int_{\Gamma} w \frac{\partial p^{\prime}}{\partial \mathbf{n}} \mathrm{d} \Gamma=-\int_{\Gamma_{a}} \frac{1}{c_{0}} w \frac{\partial p^{\prime}}{\partial t} \mathrm{~d} \Gamma .
$$

Subsequently, the computational domain is discretized using Lagrangian finite elements of first order. This allows us to set up a linear system of equations that needs to be solved in each time step in order to obtain the transient sound field.

In order to achieve good accuracy of the numerical solution, the following procedure has proven to provide the best results. First, the source term on the right-hand side of (4) is computed using the FE approach on the fine CFD grid. Then the source terms are transferred to another grid, which is better suited for the wave equation, using an energy-conserving interpolation technique. The sound emission from these sources is then computed using FEM on the acoustic grid.

\section{Experimental Setup}

The air-cooling of a generic electronic device was subjected to the investigations and simulations performed. As the interaction of the flow field with the obstacles, that is, electronic chips, pins, and heat sinks, can be a source of noise due to the production of, for example, vortex shedding or turbulent shear stresses, these geometric details are present in the experimental and numerical setup.

4.1. Generic Electronic Device. The model of the generic electronic device can be divided into four regions: the inflow area in front of the fans, the fans producing the air flow used for cooling, the settling chamber to generate an even flow through each card slot, and the card slots where the electronic components are located. In the real system, which served as a model for the generic device under investigation, the settling chamber is used to even the flow through the different card slots, especially if not all slots are in use.

The overall dimensions of the electronic device (Figure 1) are $520 \times 153 \times 338 \mathrm{~mm}$. It holds eleven card slots, each with an electronic component installed inside. The flow is generated by two axial, five-bladed $119 \mathrm{~mm}$ fans with an outer rotor diameter of $112.9 \mathrm{~mm}$. The design of the electronic components is based on real components for personal computers (i.e., network, sound, and graphic cards).

4.2. Measurements. The acoustic measurements were performed in the anechoic chamber of the University of Erlangen-Nuremberg. The generic electronic device was configured in the same way as for the simulations (Figure 1(b)): two installed fans with a rotational speed of $5000 \mathrm{rpm}$ and a settling chamber depth of $70 \mathrm{~mm}$.

Two Brüel \& Kjær Type 4189 microphones were placed at a distance of $1 \mathrm{~m}$ from the generic electronic device. The microphone facing the inlet area is aligned in the center and 


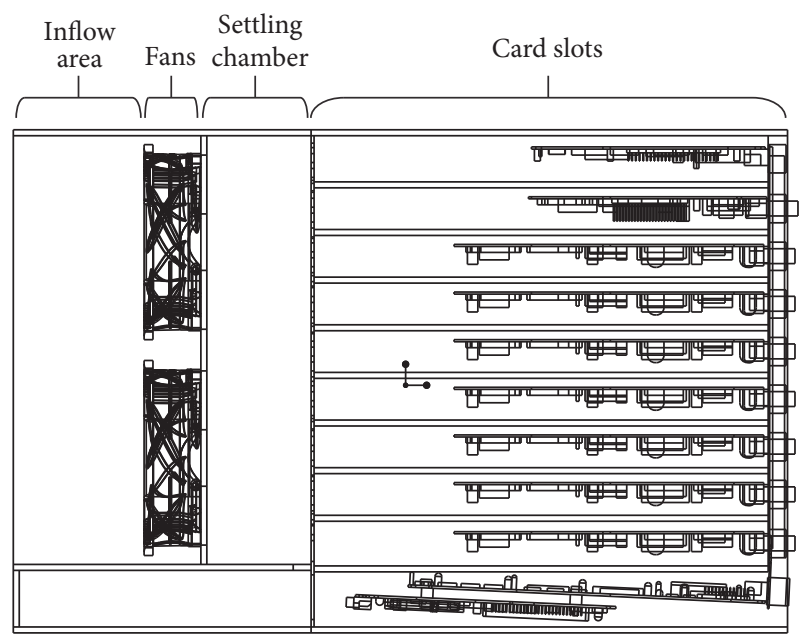

(a) Schematic drawing

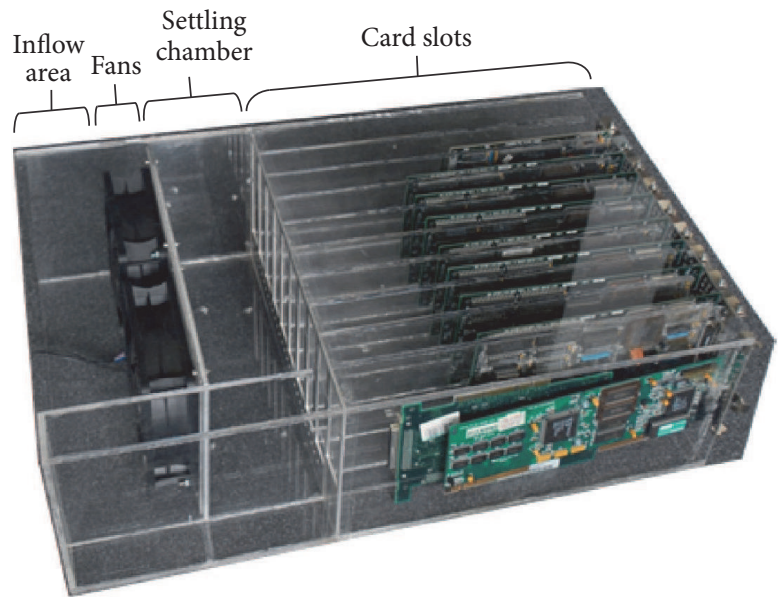

(b) Real setup

Figure 1: Generic electric device.

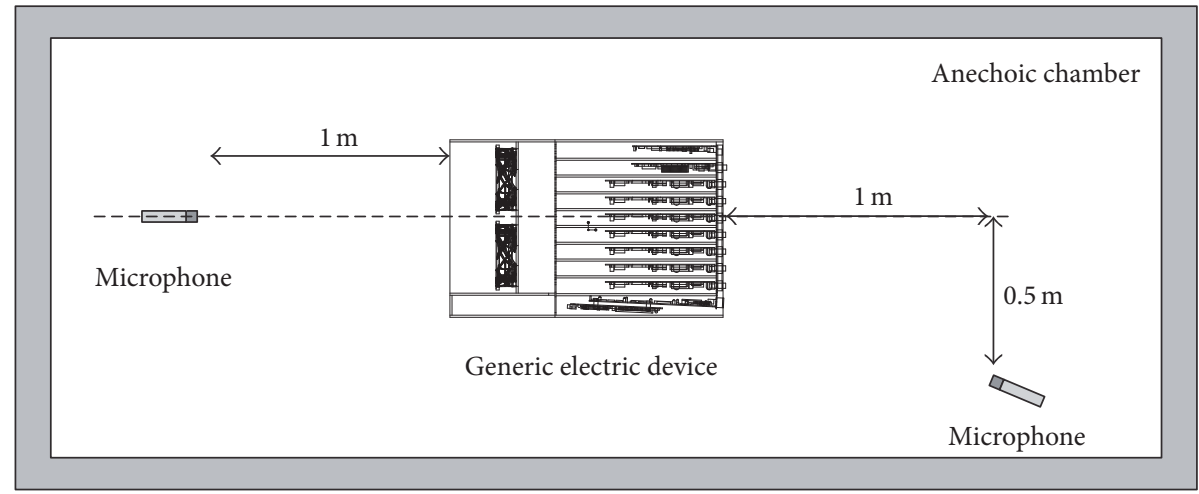

FIGURE 2: Setup for acoustic measurements.

the microphone at the outlet is installed approximately $50 \mathrm{~cm}$ eccentric to be out of the free stream generated by the air flow through the device (Figure 2). In the vertical direction, both microphones are placed at half of the device's height.

The results of the acoustic measurements were compared with the acoustic data received from the simulations. The measurements were performed with a sampling rate of $50 \mathrm{kHz}$ and a measuring time of $30 \mathrm{~s}$.

\section{Simulation Process of a Generic Electronic Device}

The first step of the simulation process was the generation of the CAD model, which served for both the CFD and acoustic mesh generation. During the CAD build process of the model, attention was paid to accommodating major electronic structures on the printed circuit boards, that is, through-hole devices and comparatively large surfacemounted devices (see Figure 3(b)). The whole generic electronic device was modeled in CAD including an additional inlet and outlet plenum. The manufacturer's original CAD data for the fan were simplified at the casing (removal of wires and reinforcing ribs) and at the hub (removal of ventilation slots for cooling of motor) to ensure proper mesh generation (Figure 3(a)). Two different meshes were generated, one for the flow simulation and the other for the acoustic simulation. For the acoustic simulation, the mesh can be coarser than the CFD mesh, because the acoustic wavelength is usually greater than the turbulent length scales of the flow.

5.1. Flow Simulation. The mesh was generated using the ANSYS Workbench Mesher. The unstructured mesh consists of tetrahedra and prism elements holding a total element number of 39.6 million and 13.4 million nodes, respectively; 9.2 million elements and 3.3 million nodes correspond to each fan as shown in Figure 4(a). The whole flow region was divided into several subregions with each one having been meshed separately and being connected to its adjacent neighbor regions using interfaces. The boundary layer within the fans has a thickness of about $1 \times 10^{-5} \mathrm{~m}$ and is resolved with 12 prism layers. The regions of the electronic cards consist, overall, of 16.1 million elements and 5.3 million nodes. Figure 4(b) shows exemplarily the mesh for one electronic card. The boundary layer on the walls of the card slots is 


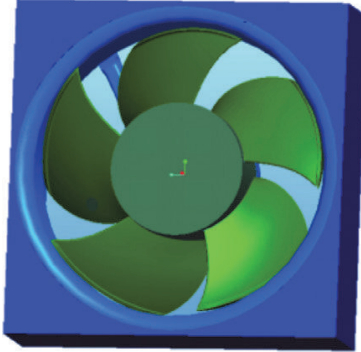

(a) Fan

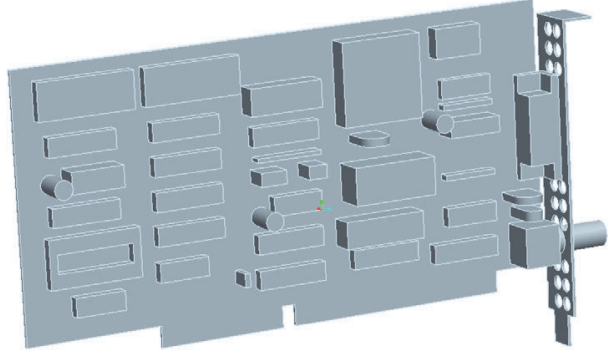

(b) Electronic component

FIGURE 3: CAD models of the components.

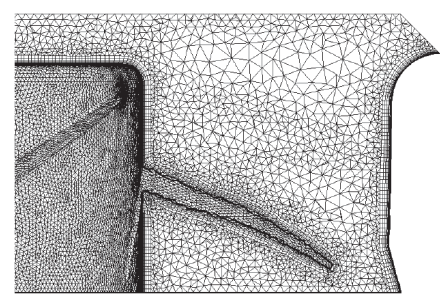

(a) Fan domain

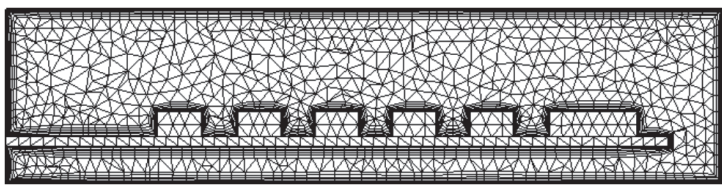

(b) Region of an electronic card

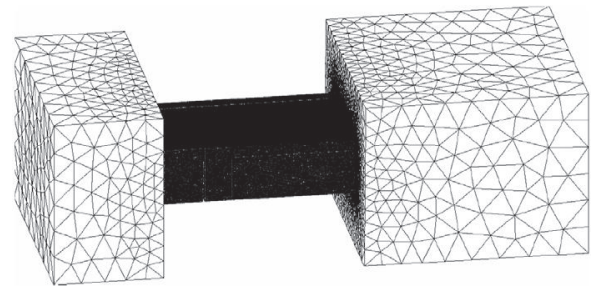

(c) Whole simulation domain including inlet and outlet plenum

Figure 4: CFD mesh.

resolved with ten layers (transition ratio: 0.5; growth rate: 1.3 ) and the electronic components on the cards are encased in seven prism layers with a total height of $2 \times 10^{-3} \mathrm{~m}$ and a growth rate of 1.3. The remaining 5.1 million elements and 1.5 million nodes belong to the inlet and outlet regions and also the settling chamber incorporating 10-12 prism layers (see Figure 4(c)).

The flow simulations were performed with two different turbulence models: the Unsteady RANS-SST model and the SAS-SST model. Both CFD simulations involve the same setup except for the turbulence models. The fans are rotating with a frequency of $5000 \mathrm{rpm}$ and are linked to the nonrotating domains, that is, the inflow region and the settling chamber (Figure 1), with a transient rotor-stator interface. The fluid maintains the characteristics of air at $25^{\circ} \mathrm{C}$ with a constant density. Owing to the low Mach number of only 0.01 at the blade tips, incompressibility of the fluid can be assumed. The CFD simulations are performed in an isothermal manner. Our preliminary experiments showed that the influence of temperature on the sound emission, at least in the temperature range that electronic devices can withstand, that is, up to $80^{\circ} \mathrm{C}$, is negligible. A mass flow condition with a value of $0.15 \mathrm{~kg} \mathrm{~s}^{-1}$ is applied to the inlet boundary. The outlet is represented by an opening boundary condition with a relative pressure of $0 \mathrm{~Pa}$. The time step size is $\Delta t=10^{-4} \mathrm{~s}$ and the number of inner iterations is set to eight.

5.2. Acoustic Simulation. The acoustic simulations were performed using the in-house FE solver CFS++ (Coupled Field Simulation in $\mathrm{C}++$ ), which is being developed jointly at the Technical University of Vienna and the University of Erlangen-Nuremberg. First, the flow-induced sound sources were computed according to (4). This operation was done on the CFD mesh in order to preserve the full spatial resolution of the flow computation. Good numerical accuracy is critical in this step; otherwise the spatial derivatives required to compute sound sources from the flow data would produce too much numerical noise. Subsequently, the sound sources were interpolated using an energy-conserving scheme onto the mesh used to compute sound propagation.

The need for a separate acoustic mesh is due to requirements in spatial resolution that are completely different compared with the flow computation. The CFD mesh needs a small cell size in boundary layers and in regions where the flow is turbulent. On the other hand, the CFD mesh can be rather coarse where the flow is laminar. In contrast, the 


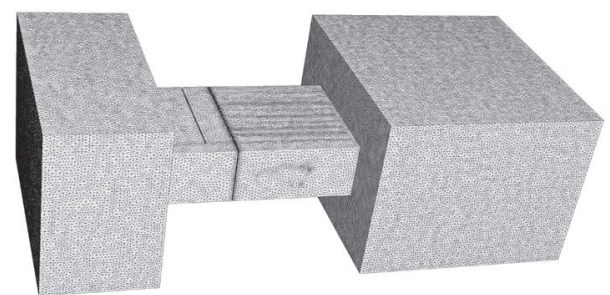

Figure 5: Acoustic mesh.

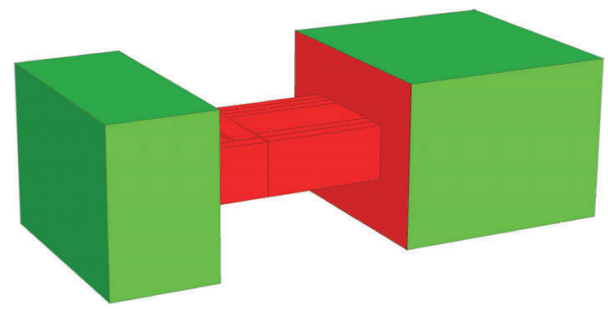

FIgURE 6: Acoustic boundary conditions: acoustically hard walls (red) and absorbing boundary (green).

acoustic mesh does not need to resolve boundary layers, but it must properly resolve the smallest wavelength in the whole domain. That is why we used an unstructured tetrahedral mesh without prism layers at rigid boundaries (Figure 5), but with an almost uniform element size. The maximum edge length of the tetrahedra was set to $8 \mathrm{~mm}$. The element size was refined only slightly at the surface of the fans and electronic components in order to account for the actual shapes of the rigid boundaries. The time step size was the same as in the flow simulation. With the Nyquist-Shannon sampling theorem and a temporal resolution of $\Delta t=1 \times 10^{-4} \mathrm{~s}$ (see Section 5.1), the maximum resolved acoustic frequency is $f_{\max }=5000 \mathrm{~Hz}$, resulting in a minimal acoustic wavelength of $\lambda_{\text {min }}=c_{0} / f_{\max }=0.069 \mathrm{~m}$ that is resolved by at least nine mesh nodes. This spatial resolution leads to a mesh size of three million elements with 621000 nodes. Approximately 93000 elements with 24000 nodes are located in each fan.

The acoustic simulation was performed with the same fluid properties as the flow simulation, that is, air at $25^{\circ} \mathrm{C}$. To avoid nonphysical reflections of the acoustic waves, absorbing boundary conditions were applied to the inlet and outlet plenum on the same surfaces as the inlet and outlet boundary conditions of the flow simulation. The surfaces of the electronic device's housing and the particular surfaces of the inlet and outlet plenum contacting the housing were acoustically hard walls (Figure 6).

A frozen rotor model was used for the axial fans in the acoustic simulation. Furthermore, the simulation domain does not extend to either of the microphone positions, so the sound field had to be extrapolated to the microphone positions. This is allowed provided that the near field around the device is fully contained in the simulation domain. This means that the absorbing boundaries are in the far field and the sound pressure on these boundaries can be extrapolated to arbitrary points outside the simulation domain using

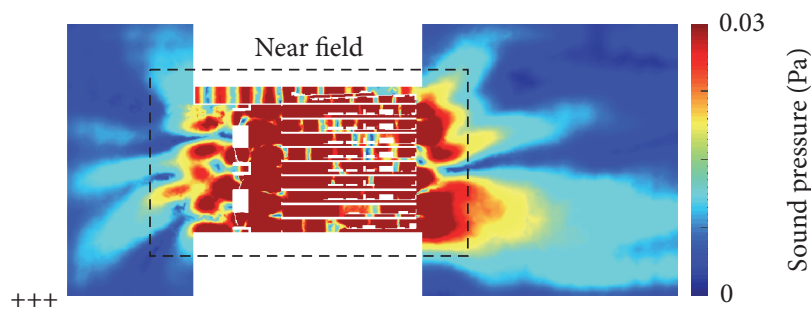

FIGURE 7: Time-harmonic magnitude of sound pressure generated by a monofrequent sound source at $4.0 \mathrm{kHz}$; shown is the crosssection through the axes of both fans.

the inverse distance rule. As a rough approximation for the stretch of the device's near field we used the equation for the near-field length of a baffled piston, namely, $N=$ $D^{2} / 4 \lambda_{\min }=D^{2} f_{\max } / 4 c_{0}$. Thereby, $D=33 \mathrm{~cm}$ is the length of the longer edge of the device's rectangular inlet opening and serves as the characteristic size in the baffled-piston model. Strictly speaking, this formula is only valid for circular pistons. However, we can obtain a worst-case approximation of the near-field length by using the longer edge for the characteristic length $D$ and the maximum frequency. With the size of the inlet plenum as the maximum near-field length $N_{\text {max }}=28 \mathrm{~cm}$, we determined the maximum frequency that allows for extrapolation to be $f_{\max }=4 c_{0} N_{\max } D^{-2} \approx$ $3.6 \mathrm{kHz}$. This approximation was confirmed by a preliminary simulation with an artificial sound source inside the device radiating sine waves at $4.0 \mathrm{kHz}$ (cf. Figure 7).

During the first $30 \mathrm{~ms}$ of the acoustic simulation, the sound sources were tapered with a quadratic sine function in order to achieve a fade-in of the sources. The reason for this is that sound sources were computed from the flow only after the flow through the device had fully developed its turbulent structure. Instantaneously applying the full-scale sources to the initially quiescent acoustic medium would result in numerical acoustic waves that can have an adverse effect on the real physical solution [17]. This effect can be avoided using a fade-in of the sound sources. For time stepping a second order Newmark scheme was used.

A comparison of two different boundary conditions, namely, an ABC and a PML, was performed. An artificial point source was placed within the device and the sound pressure with an absorbing boundary condition (ABC) and a perfectly matching layer (PML) was calculated with a harmonic analysis in the range from 10 to $2000 \mathrm{~Hz}$ in $10 \mathrm{~Hz}$ steps. Figure 8 shows the difference of simulated sound pressure for $\mathrm{ABC}$ and PML relative to the source strength $s$ :

$$
\operatorname{err}(f)=\frac{\left|p_{\mathrm{ABC}}(f)-p_{\mathrm{PML}}(f)\right|}{s}
$$

The frequency range from 200 to $350 \mathrm{~Hz}$ shows the largest differences between ABC and PML boundary condition. For frequencies larger than $500 \mathrm{~Hz}$ almost no difference can be found. Below $500 \mathrm{~Hz}$ we observe that the ABC cannot fully damp out acoustic modes that are purely artificial and arise from using a finite-size computational domain to model an unbounded domain. However, we can filter this spurious 


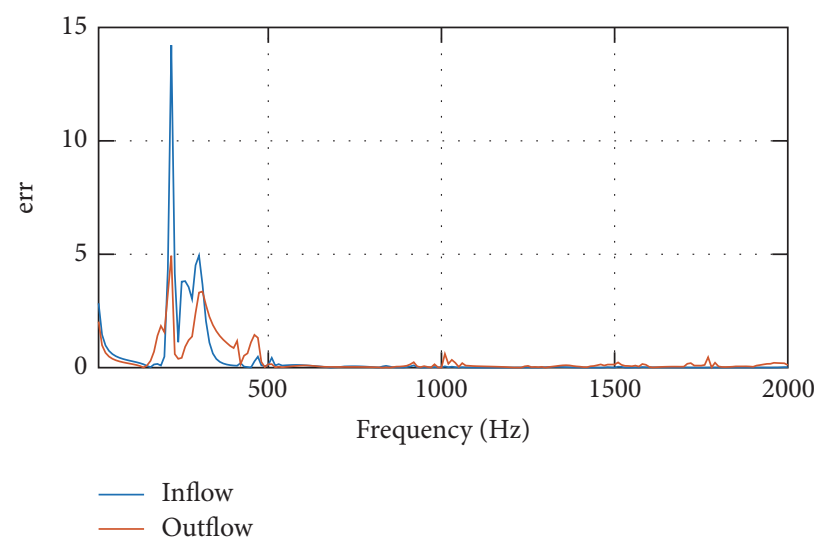

FIgURE 8: Comparison of ABC and PML boundary condition.

effect from the results of the simulation using $\mathrm{ABC}$, because it occurs at discrete frequencies only. Despite the differences between the ABC and PML boundary conditions an ABC was chosen due to much smaller computational efforts of the $\mathrm{ABC}$ over the PML.

In addition to the sound propagation simulations based on the aeroacoustic sources, the acoustic eigenmodes were calculated using the same acoustic setup. These results will be used to identify peaks in the acoustic spectra that are not triggered by obvious effects like blade passing.

5.3. Structural Simulation. As the results of the acoustic simulations will only include aeroacoustic sources but are compared to measurements, which also include structureborne sound, an additional structural modal analysis has been carried out. The goal of this finite element simulation was to identify structural eigenmodes contributing to the noise emission which are present in the measurements but not in the CAA simulations.

\section{Results}

6.1. Structural Eigenmodes. Only the first two eigenmodes were identified as their eigenfrequencies are already higher than the acoustic frequency range of the simulations. Figure 9 depicts both eigenmodes. The first eigenmode shows a vibration at the inlet section of the electronic device with an eigenfrequency of $2320 \mathrm{~Hz}$. The second eigenmode causes a deflection of the mainboard area at the outlet with an eigenfrequency of $2484 \mathrm{~Hz}$. Consequently, no comparison errors of acoustic simulation and measurement are to be expected due to structural eigenmodes in a range up to $2 \mathrm{kHz}$.

6.2. Flow Simulation. The simulation time of the flow field was $0.21 \mathrm{~s}$ with 2100 time steps, in the case of the URANSSST, and $0.64 \mathrm{~s}$ with 6400 steps in case of the SAS-SST. The flow field was first initialized with a steady-state simulation; then the setup was changed to the unsteady case. Before exporting the flow data, the flow field was allowed to adapt to the unsteady conditions for approximately $0.2 \mathrm{~s}$. The exported quantities are the hydrodynamic pressure and the three velocity components. The URANS simulation took 115,200 CPU hours to complete (including initialization) and the SAS simulation 157,000 hours. The calculations were performed at the HPC center of the University of ErlangenNuremberg.

The time step size of $1 \times 10^{-4} \mathrm{~s}$ in combination with the fine spatial resolution of the fan domains results in an average CFL number of 3.5 for the fan domains. While the highest values are to be found in the blade tip gap, the values in the boundary layer of the blades vary between 3 and 8 with a decrease in direction to lower perimeters. A lower CFL number can be achieved by further decreasing the time step size. Figure 10 holds a distribution of the CFL number in a cut plane through both fan axes and in a cut plane perpendicular to one fan axis of the URANS simulation.

Figures 11 and 12 show the velocity and the pressure field on a cut plane through the axis of the fans for the URANS-SST and SAS-SST simulations. A comparison of these two figures demonstrates the advantages of the SAS model: the resolved scales are finer and represent the flow field in more detail. Accordingly, the fluctuations incorporate higher amplitudes at higher frequencies in the SAS-SST simulation compared with the URANS-SST simulation. This is important as the frequency range of the calculated acoustic source terms is based on the fluctuations existing in the flow field. The fact that the SAS model is less modeling but more resolving with respect to the turbulent structures is underlined by Figure 13, which highlights the decrease in the eddy viscosity.

Optimizations of the air-cooling can be derived from the flow field plots. The card slots that are placed in line with the hub receive lower flow rates and, correspondingly, less cooling. Depending on the inflow angle into the card slots, separation areas form due to detachment of the flow, as depicted in Figures 11(a) and 12(a). The implementation of baffle plates would lead to more homogeneous inflow conditions with smaller inflow angles and hence less separation.

The $Q$-criterion is a common method for visualizing vortex regions. It is defined as the second invariant of the velocity gradient tensor. Dominant vortex regions (Figure 14) can be found behind fans, in the inflow region of the card slots and at the electronic parts soldered onto the printed circuit boards.

The relative velocity and pressure distribution in Figure 15 around the fan blade depicts a flow separation area close to the leading edge on the suction side. Due to the high rotational speeds of $5000 \mathrm{rpm}$ in combination with large angle of attack the flow detaches from the blade. This causes vortex shedding in the blade wake, demonstrated by some eddies located close to the trailing edge in the pressure plot. Additionally, a stagnation point at the leading edge can be identified which corresponds with a pressure maximum.

A comparison of the hydraulic pressure fluctuations for six different positions on the blade surface is shown in Figure 16. The SAS model introduces less damping than the URANS model and thus leads to a slower decay of the fluctuations. Both models produce dominant peaks at the blade passing frequency and all harmonics. But the SAS model is also capable of capturing fluctuations in between 


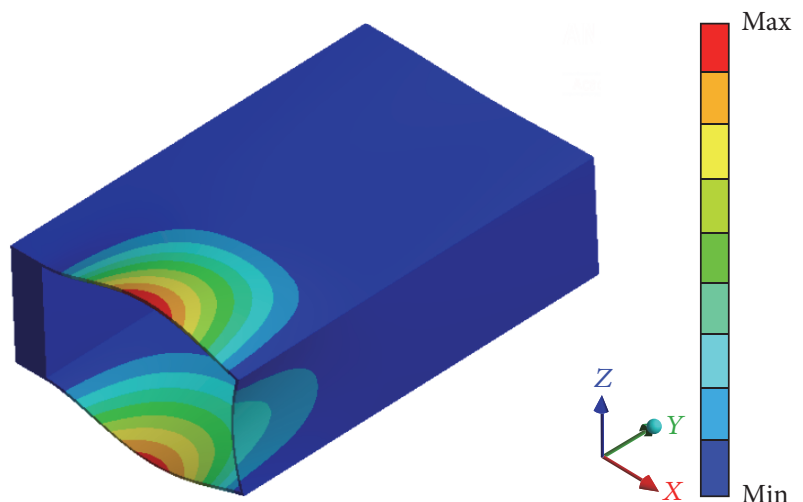

(a) At $2320 \mathrm{~Hz}$; looking at the inflow area

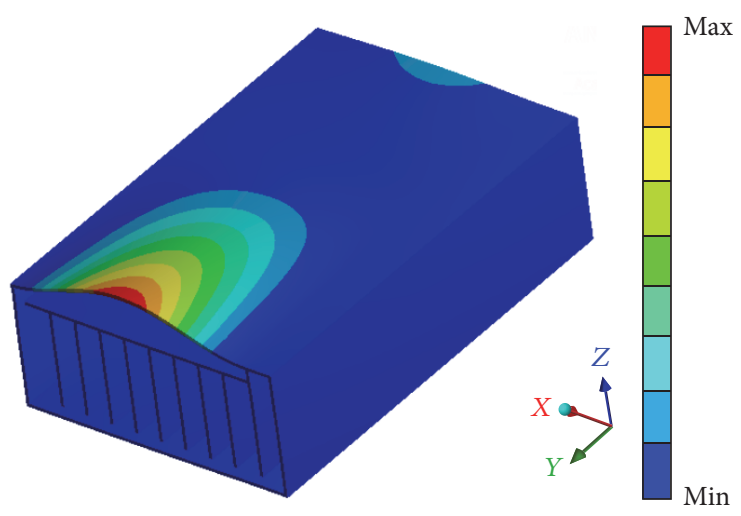

(b) At $2484 \mathrm{~Hz}$; looking at the outflow area

FIGURE 9: Structural eigenmodes (displacement shown).

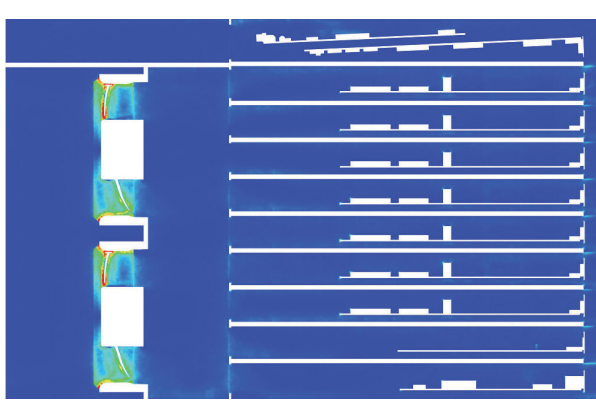

(a)

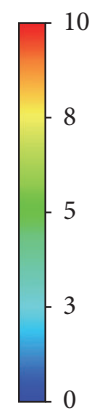

0

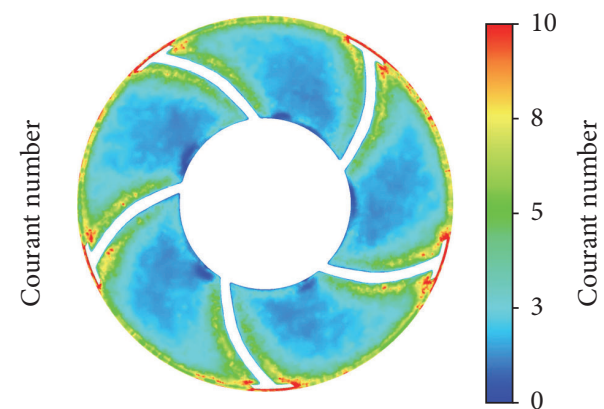

(b)

FIGURE 10: CFL number on a cut plane through both fan axes (a) and perpendicular to a fan axis (b).

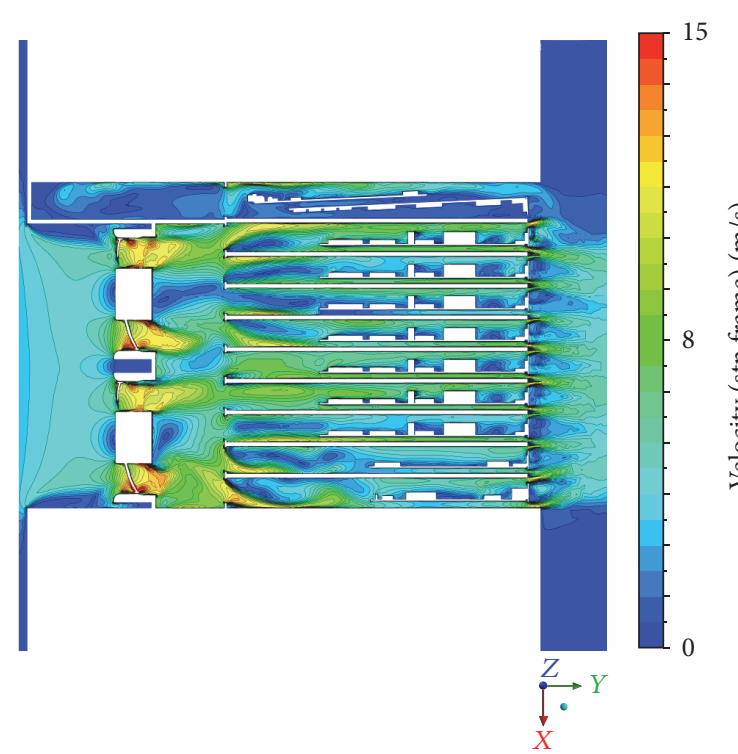

(a) Velocity field

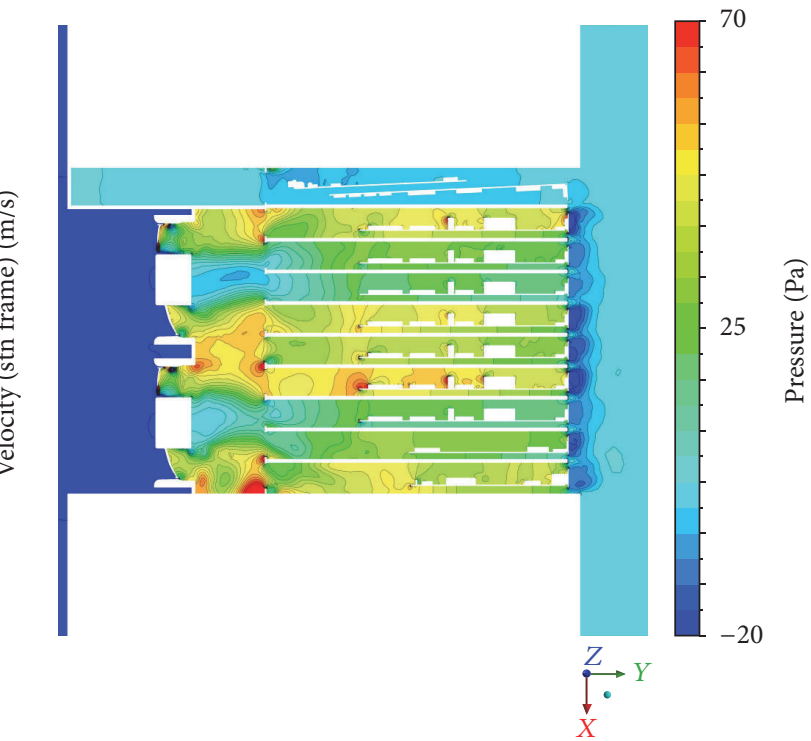

(b) Pressure field

FIGURE 11: URANS simulation. 


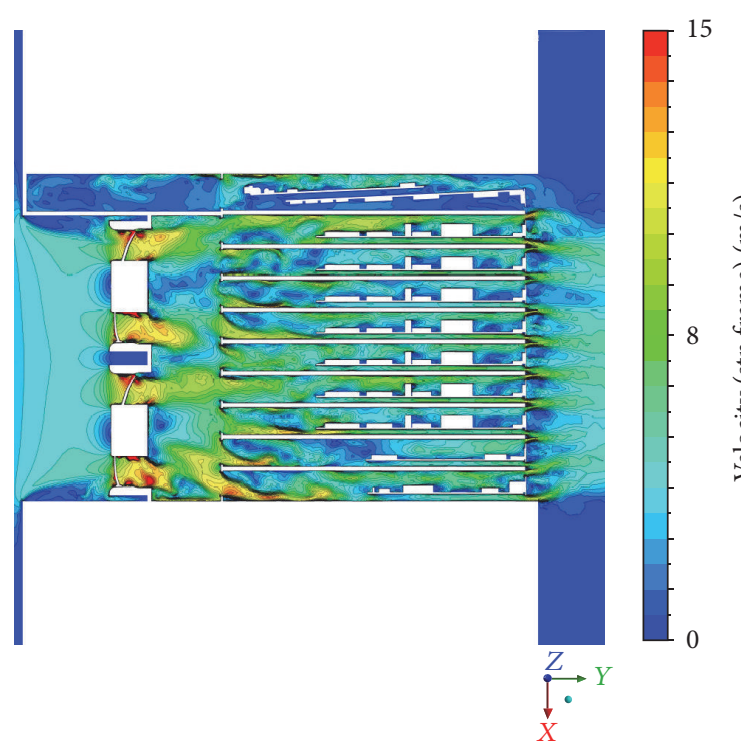

(a) Velocity field

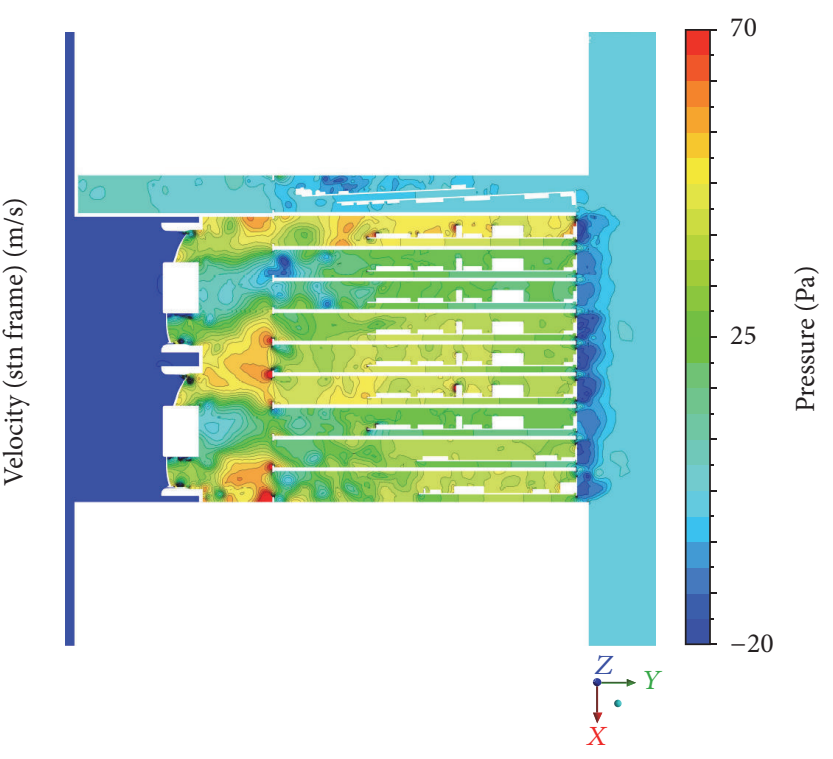

(b) Pressure field

FIGURE 12: SAS simulation.

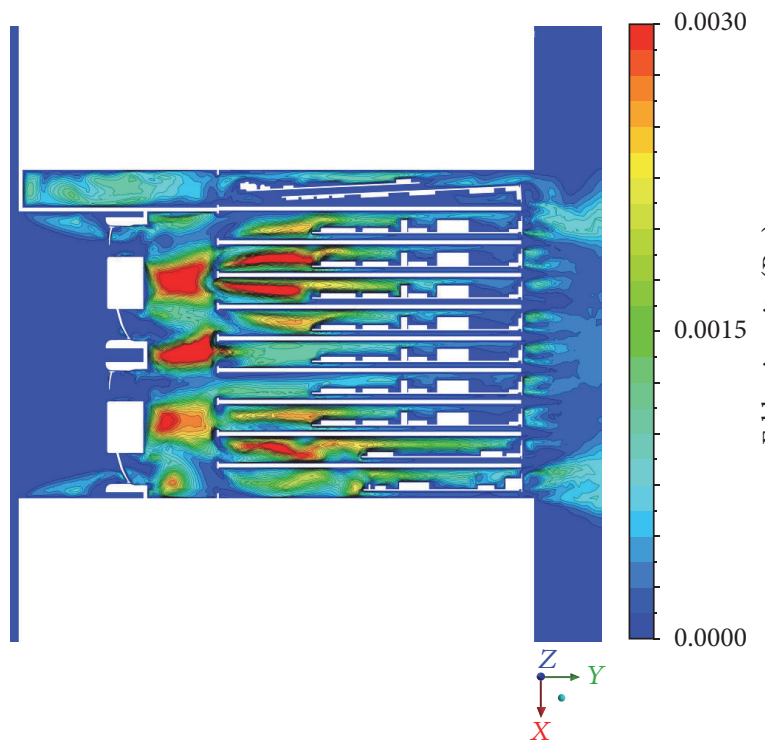

(a) URANS

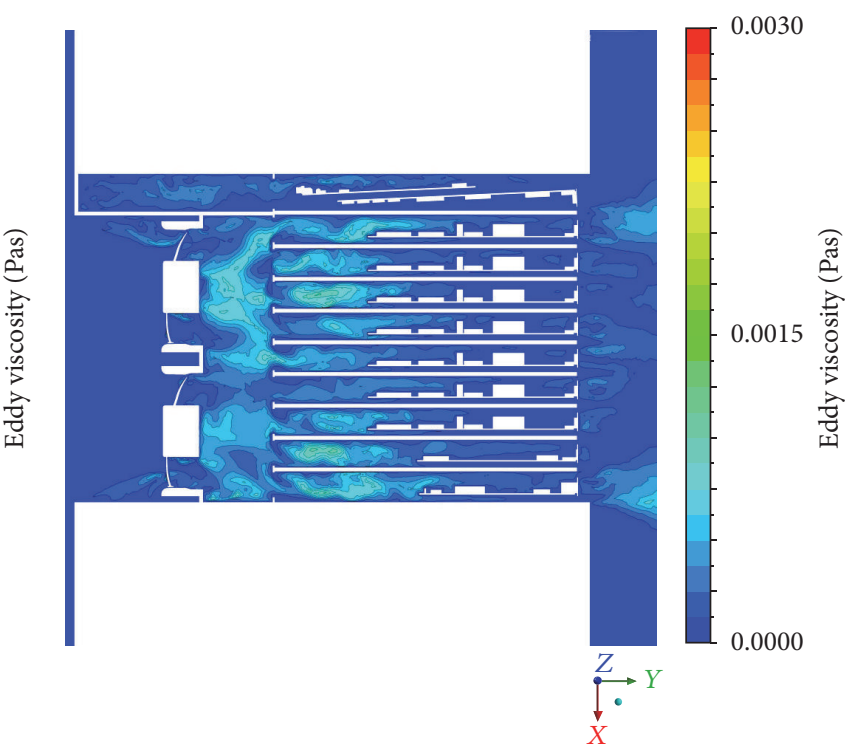

(b) SAS

FIGURE 13: Eddy viscosity.

these dominant effects, resulting in higher pressure amplitudes. Also some of the effects explained in Table 1 can be found in the pressure spectrum of the trailing edge point. Particularly rotor-stator and blade-stator interaction results in an increase of the pressure amplitudes. In general, the leading edge is exposed to the highest fluctuations as it first cuts the incoming eddies.

The mean velocity downstream of the fans is plotted in Figure 17. Both turbulence models lead to very similar trends. The SAS model generates a slightly more detailed velocity profile in the downstream blade wake in regions of high velocity gradients. The velocity profiles smoothen with an increasing distance to the fans due to the momentum transfer in the fluid.

Likewise, the spatial pressure distribution is shown in Figure 18. Here, SAS and URANS produce very similar spatial distributions and lead to the same rise in pressure with an increasing distance to the fans as the dynamic pressure component is converted into the static one.

While the time-averaged pressure results in no significant differences between the URANS and SAS models, the advantages of the SAS model become obvious for the wall pressure spectra given in Figure 19 being in accordance with Figure 16. The SAS model is much better resolving pressure 


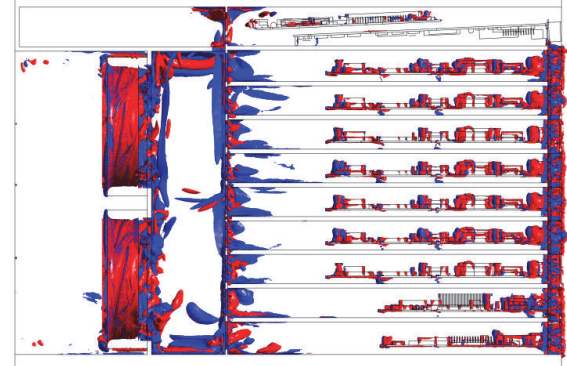

(a) URANS

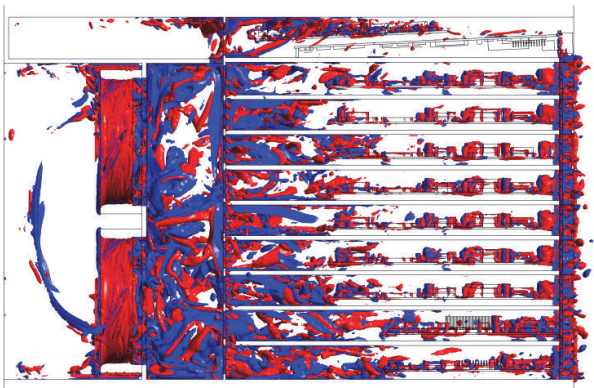

(b) SAS

Figure 14: Q-criterion on isosurfaces of $\pm 3 \times 10^{5} \mathrm{~s}^{-2}$ (blue: $-3 \times 10^{5} \mathrm{~s}^{-2}$; red: $+3 \times 10^{5} \mathrm{~s}^{-2}$ ).

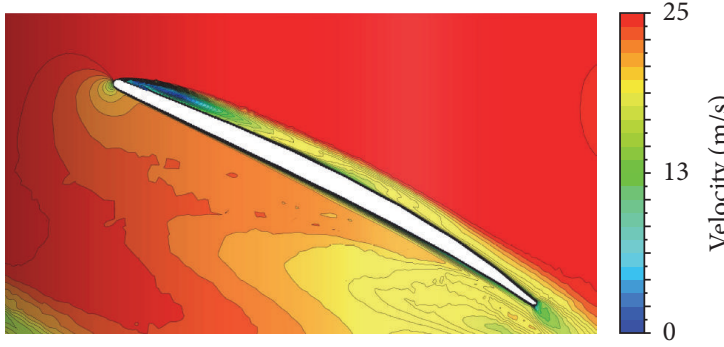

(a) Relative velocity

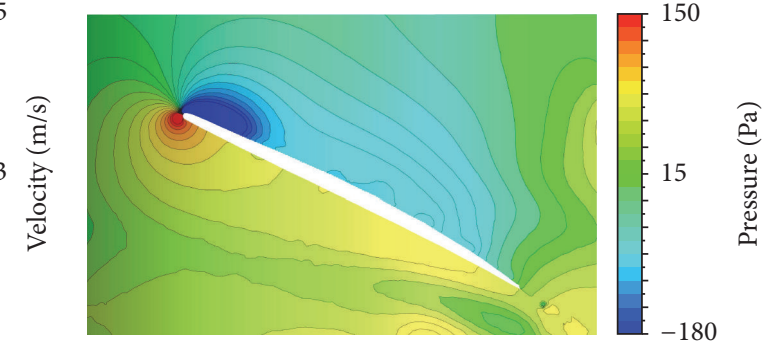

(b) Pressure

FIGURE 15: Relative velocity and pressure distribution on a cylindrical plane with $D_{\text {Plane }}=0.84 D$ for SAS simulation.

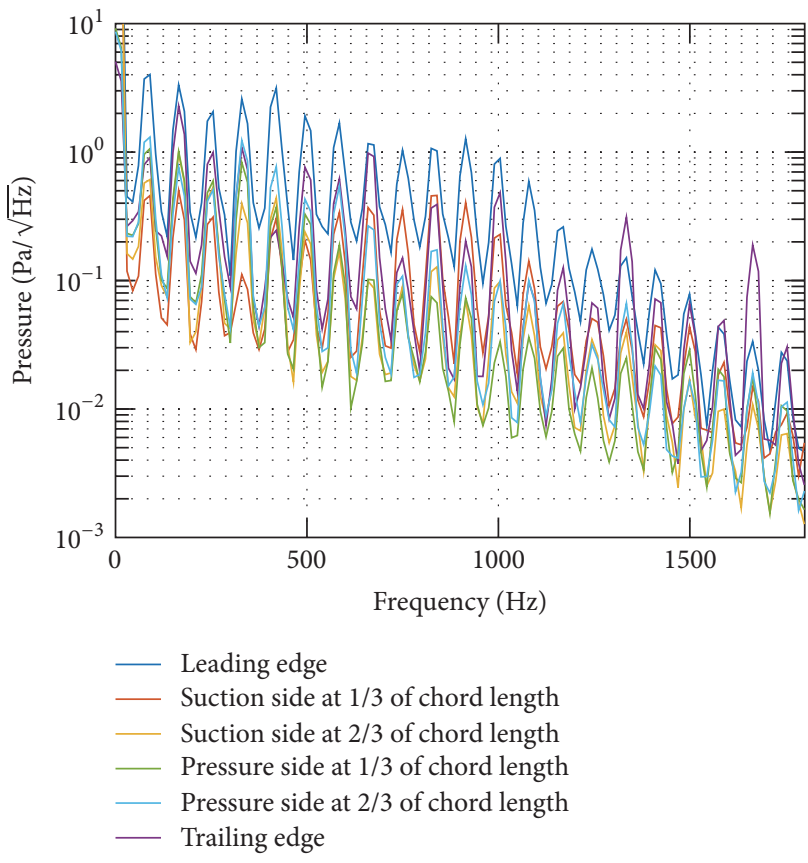

(a) URANS

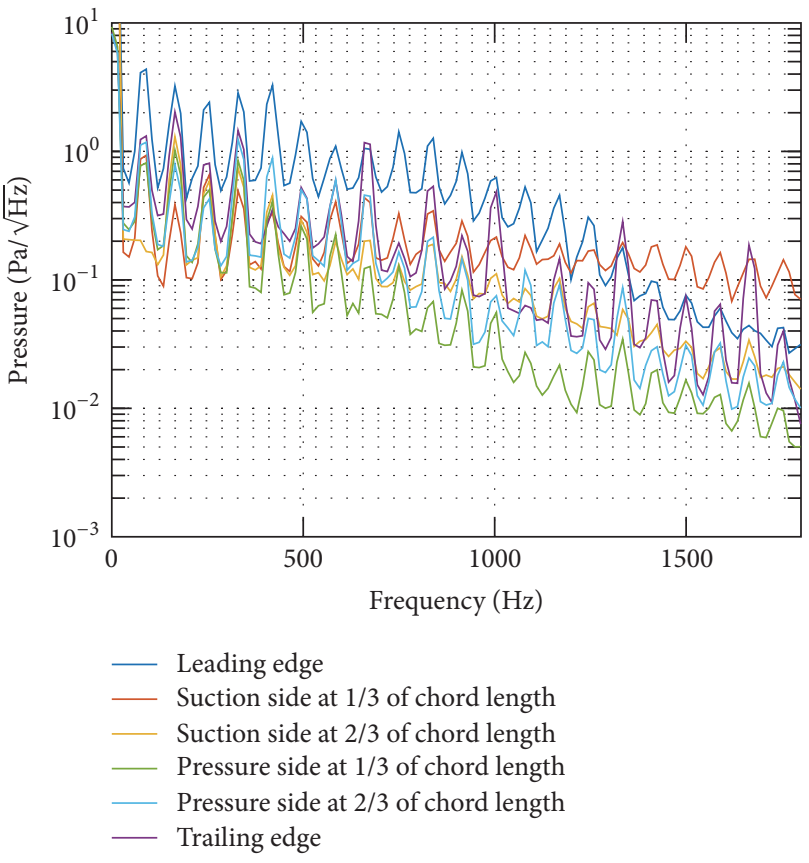

(b) SAS

FIGURE 16: Fourier transform of hydraulic pressure at six different positions on the blade surface at a diameter of 0.84D: leading edge, suction side at $1 / 3$ of chord length, suction side at $2 / 3$ of chord length, pressure side at $1 / 3$ of chord length, pressure side at $2 / 3$ of chord length, and trailing edge. 


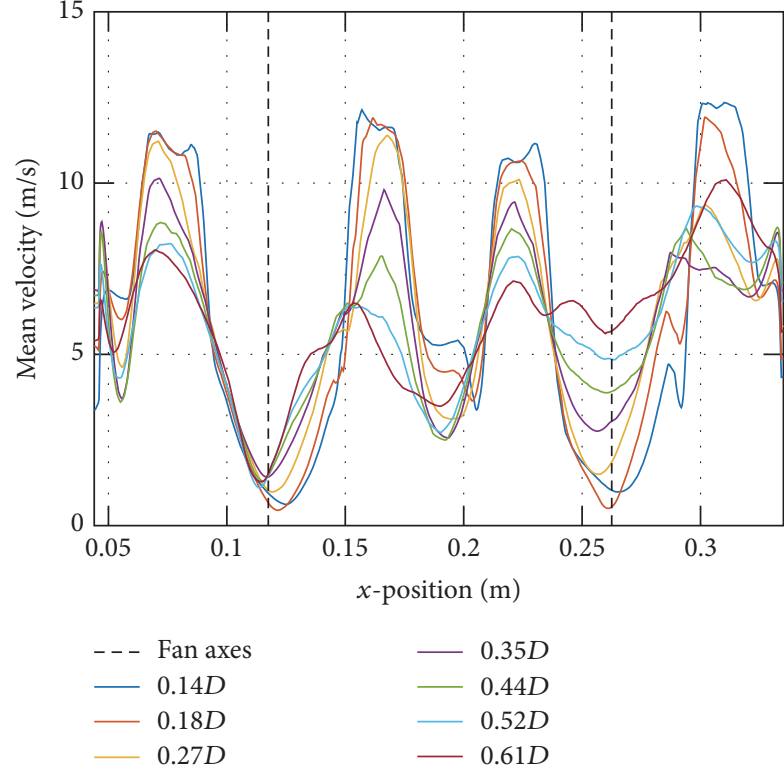

(a) URANS

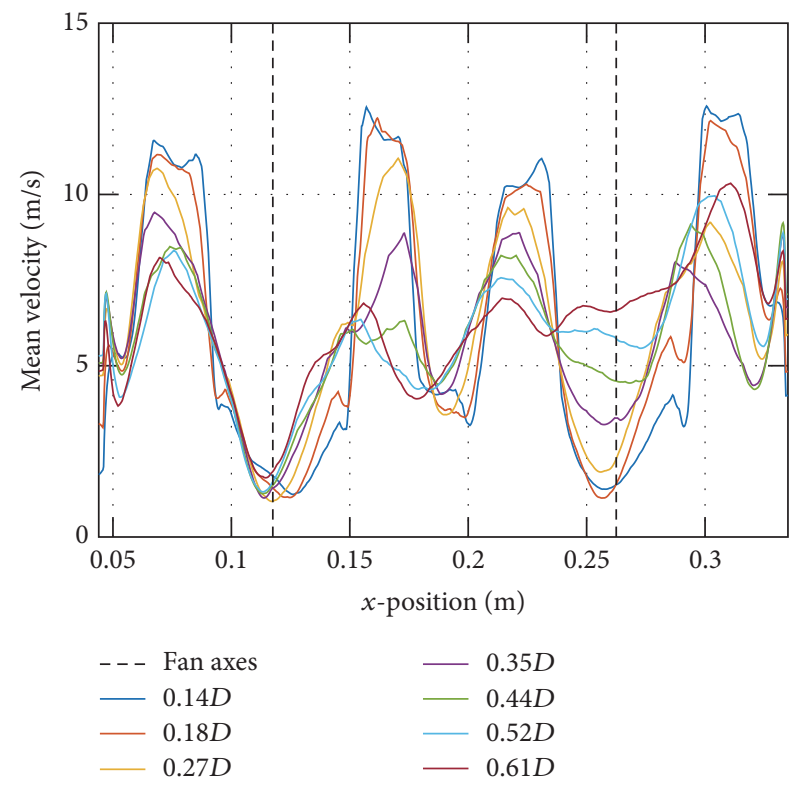

(b) SAS

FIGURE 17: Mean velocity profiles along multiple axes perpendicular to both fan axes at distances of $0.14 D, 0.18 D, 0.27 D, 0.35 D, 0.44 D$, $0.52 D$, and $0.61 D$ to the fan outlet for URANS and SAS simulation.

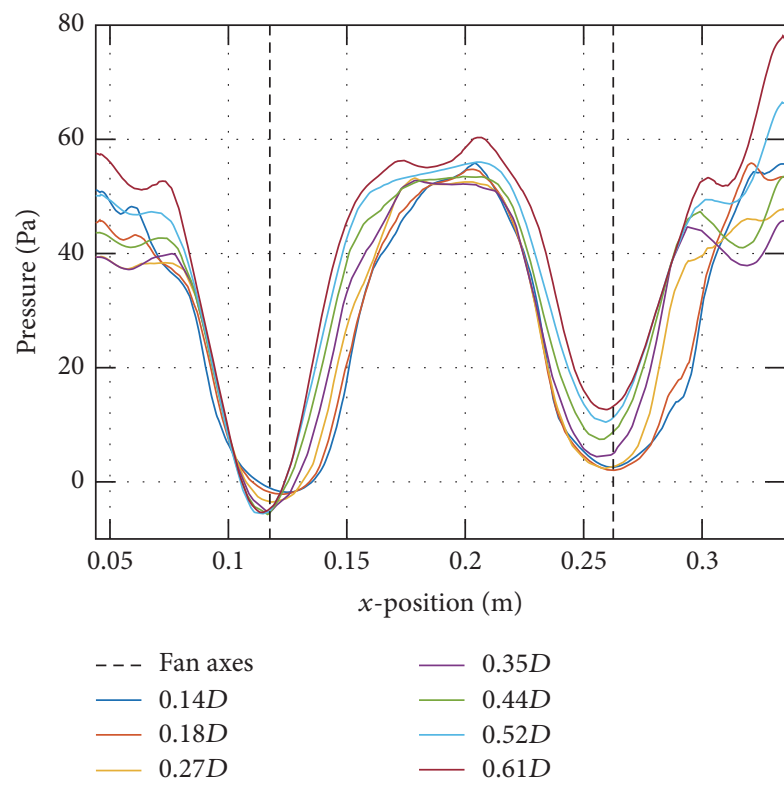

(a) URANS

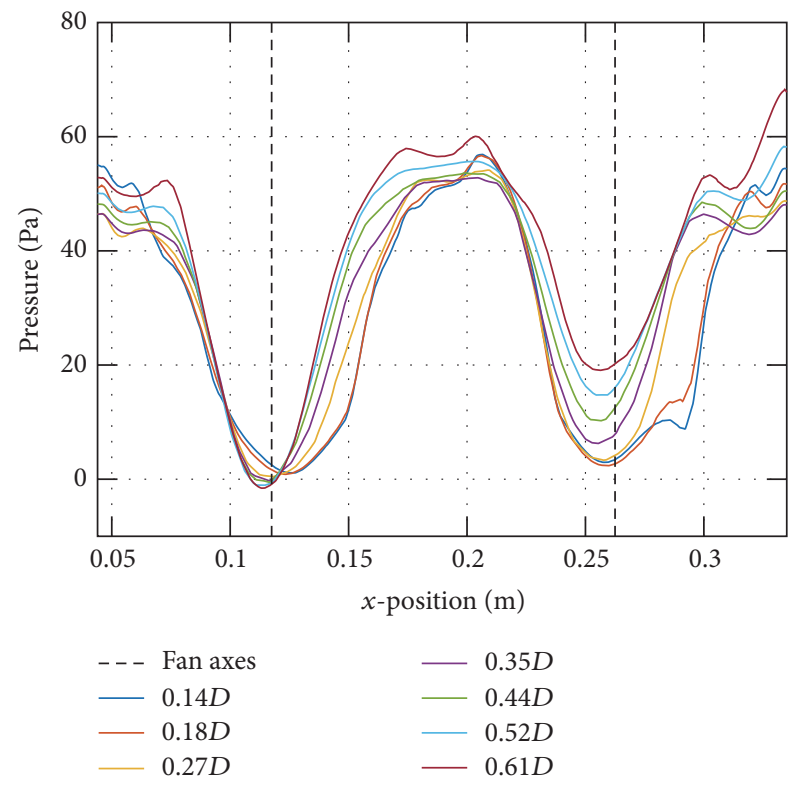

(b) SAS

FIGURE 18: Mean pressure profiles along multiple axes perpendicular to both fan axes at distances of $0.14 D, 0.18 D, 0.27 D, 0.35 D, 0.44 D$, $0.52 D$, and $0.61 D$ to the fan outlet for URANS and SAS simulation.

fluctuations generated by random turbulence in contrast to triggered phenomena like blade passing. For the SAS model the fluctuations are approximately one to two orders of magnitude higher than for the URANS model.

The damping of the URANS model can be best demonstrated by looking at the Fourier transform of the turbulent kinetic energy in Figure 20. First of all, the turbulent kinetic energy contained within the flow is higher for the SAS model, especially at higher frequencies. Secondly, the damping of turbulent eddies is much lower for an increasing downstream distance. With the SAS model the four observed positions show a decrease of less than one order of magnitude while the URANS model reduces the turbulent kinetic energy by four orders of magnitude. 


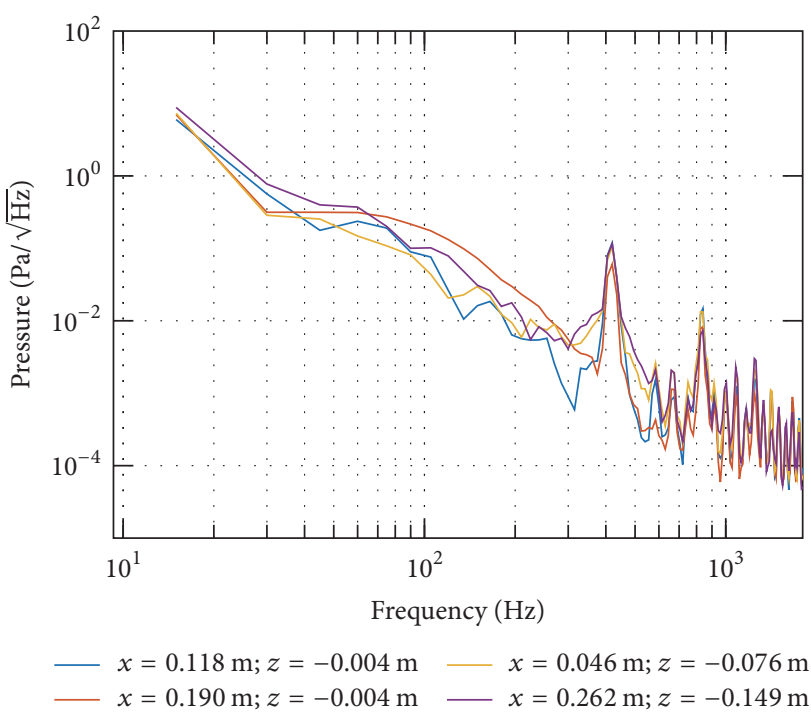

(a) URANS

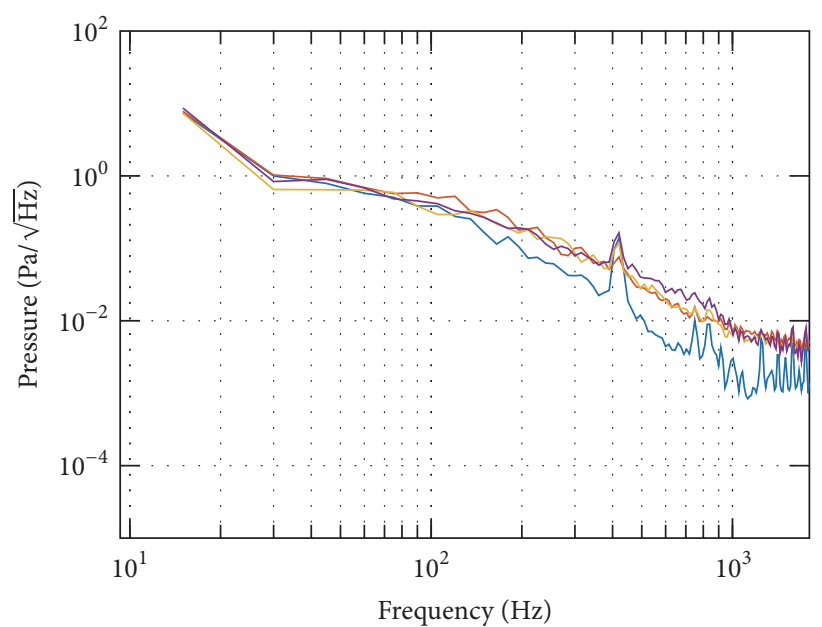

$x=0.118 \mathrm{~m} ; z=-0.004 \mathrm{~m}-x=0.046 \mathrm{~m} ; z=-0.076 \mathrm{~m}$
$-x=0.190 \mathrm{~m} ; z=-0.004 \mathrm{~m}-x=0.262 \mathrm{~m} ; z=-0.149 \mathrm{~m}$

(b) SAS

Figure 19: Fourier transform of hydraulic wall pressure $0.5 D$ behind the fan at four different positions $x=0.118 \mathrm{~m} ; z=-0.004 \mathrm{~m}, x=$ $0.190 \mathrm{~m} ; z=-0.004 \mathrm{~m}, x=0.046 \mathrm{~m} ; z=-0.076 \mathrm{~m}, x=0.262 \mathrm{~m} ; z=-0.149 \mathrm{~m}$ for URANS and SAS simulation.

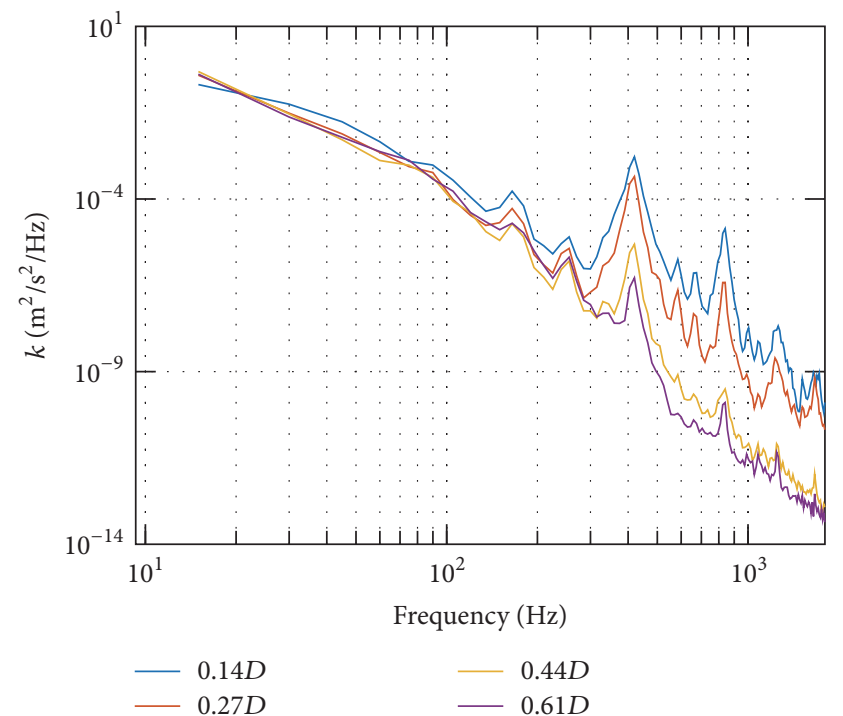

(a) URANS

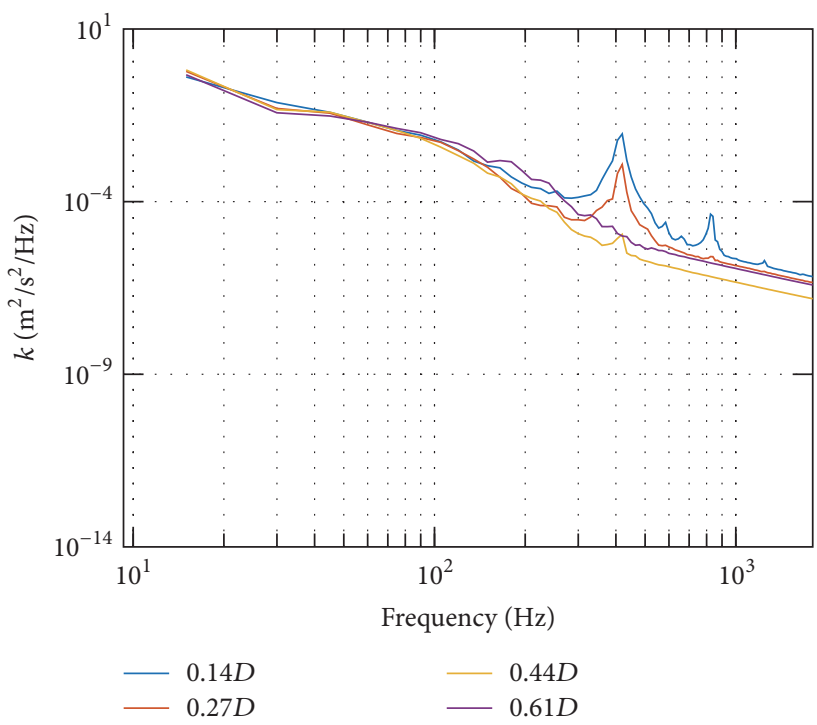

(b) SAS

FIGURE 20: Fourier transform of turbulent kinetic energy $k$ in a plane containing both fan axes at diameter of $0.75 D$ from one fan axis at four different positions downstream $0.14 D, 0.27 D, 0.44 D$, and $0.61 D$ of the fan outlet for URANS and SAS simulation.

6.3. Acoustics. In a first step, the aeroacoustic sound sources were computed and are displayed in Figure 21. While there exist acoustic formulations depending on the velocity the authors used the $\Delta p$ formulation of the aeroacoustic sources. This formulation can be directly translated into the velocity formulation shown in (2).

As one would expect, the greatest sources are found inside the fans and in their wake. We find additional sources in the separation zones, where the flow enters the card slots. Sound sources are also generated by turbulence in the wake of the electronic cards' brackets. However, the components on the electronic cards do not generate enough turbulence to transform into relevant sound sources. The difference between the sound sources obtained from the URANS and the SAS computations corresponds to the differences in the vortex structures observed in the two flow simulations through the Q-criterion (Figure 14). The SAS resolves finer vortex structures than the URANS simulation, leading to considerably greater sound sources in the entry regions of the card slots in the SAS case compared with the URANS case. Hence, the Q-criterion is a useful indicator to locate the generation of aeroacoustic sources from CFD data for 


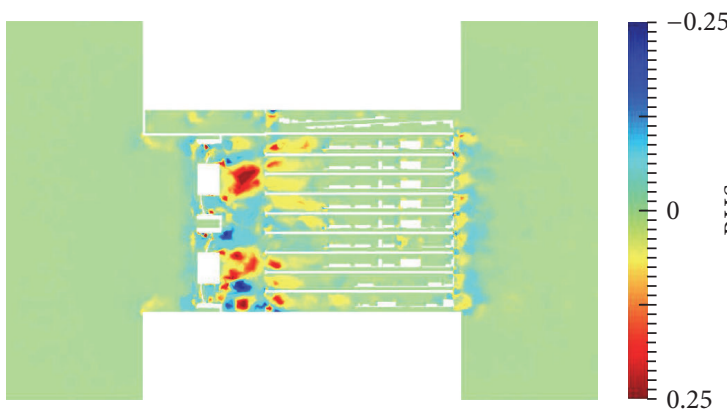

(a) Based on URANS data

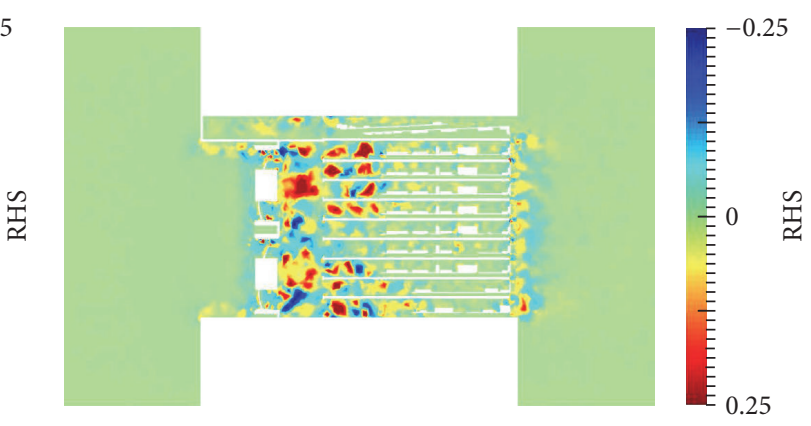

(b) Based on SAS data

FIGURE 21: Finite element representation of aeroacoustic sources computed according to the right-hand side of (4); shown is the cross-section through the axes of both fans.

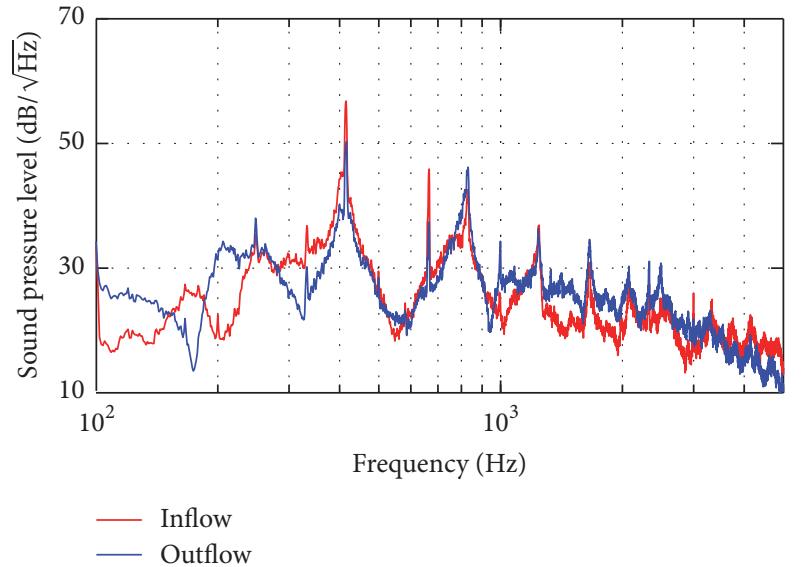

FIGURE 22: Measured sound pressure level at inflow and outflow position.

incompressible flows as it is proportional to the Lighthill sources.

The results of the acoustic measurements are presented in Figure 22 in a frequency range up to $10 \mathrm{kHz}$ and are compared to the acoustic simulations in Figure 23. The fast Fourier transformations were performed with a $1 \mathrm{~s}$-Hanning window for the measurement data and a $0.0667 \mathrm{~s}-\mathrm{Hanning}$ window for the simulation data, resulting in frequency resolution of $1 \mathrm{~Hz}$ and $15 \mathrm{~Hz}$, respectively. The acoustic measurements are shown to explain some of the higher frequent phenomena that are not present in the acoustic simulations due to the lower temporal resolution.

Figure 23 illustrates the acoustic spectra of the simulation and measurements at the inflow and outflow positions (see Figure 2). At a frequency of $\approx 4800 \mathrm{~Hz}$ as the spectra approach the Nyquist frequency, which computes as $(2 \cdot \Delta t)^{-1}=(2 \times$ $\left.10^{-4} \mathrm{~s}\right)^{-1}=5000 \mathrm{~Hz}$ and is not shown in the spectra, the amplitudes decrease quickly due to an insufficient temporal resolution. While the simulations provide data up to this frequency, only a range up to $1 \mathrm{kHz}$ should be used for evaluations at which the acoustic waves are resolved with 10 points in the temporal domain and 42 points in the spatial domain. This discrepancy in temporal and spatial resolution can be avoided by decreasing the time step to 2$2.5 \times 10^{-5}$ s resulting in an equal resolution in time and space. For the current simulation cases, damping due to insufficient temporal resolution is to be expected for frequencies higher than $1 \mathrm{kHz}$.

Supplementary acoustic and mechanical modal analyses were performed to aid explaining some of the effects observed in measurements and CAA simulations. Thereby, the first significant peak in the spectrum recorded at the outflow position of the device occurs around $215 \mathrm{~Hz}$ and can be attributed to an acoustic cavity resonance in the front panel slot (Figure 24(a)). The reason why this peak is only present at the outflow side is that the front panel slot is sealed at the inflow side. The next peak at $278 \mathrm{~Hz}$ is caused by an acoustic eigenmode that spans the settling chamber and all of the card slots (Figure 24(b)). The peaks at 417 and $833 \mathrm{~Hz}$ can be identified as the blade passing frequency, $f_{\mathrm{bp}}=$ $f \cdot z=83.3 \mathrm{~Hz} \cdot 5=417 \mathrm{~Hz}$, and its second harmonic. The prominence of the blade passing frequency and its first harmonic over the broadband background noise can be explained by its proximity to acoustic cavity resonances at 403, 416, and $868 \mathrm{~Hz}$ that occur in the card slots (Figure 24(c)) and the mainboard compartment (Figure 24(d)), respectively. This effect can even be proved by performing a discrete Fourier transform on the transient sound field obtained from the acoustic simulation. From the results of the Fourier transform, we pick the spectral line closest to the eigenmodes (Figure 25). Just by visual comparison with the eigenmodes depicted in Figures 24(c), 24(d), and 24(e) one can prove that the eigenmodes are actually excited by the sound originating from the fans. A close-up look at the blade reveals the generation of acoustic source at the leading edge and the wake (Figure 26). The peaks at 333, 667, and $1333 \mathrm{~Hz}$ correspond to the rotation-triggered interaction of the four-strutted stator with the rotor. This generates strong acoustic sources and sound pressure levels between the area of the blades and the struts as shown in Figure 27. Compared with the blade passing frequency, this phenomenon is not triggered by the number of blades. Rotor-stator interaction triggered by the blades is located at a frequency of $4 \cdot 5 \cdot 83.3 \mathrm{~Hz}=1666 \mathrm{~Hz}$ and yields another increase in the sound emission. The local maxima at approximately 2330 and $2490 \mathrm{~Hz}$ belong to the 


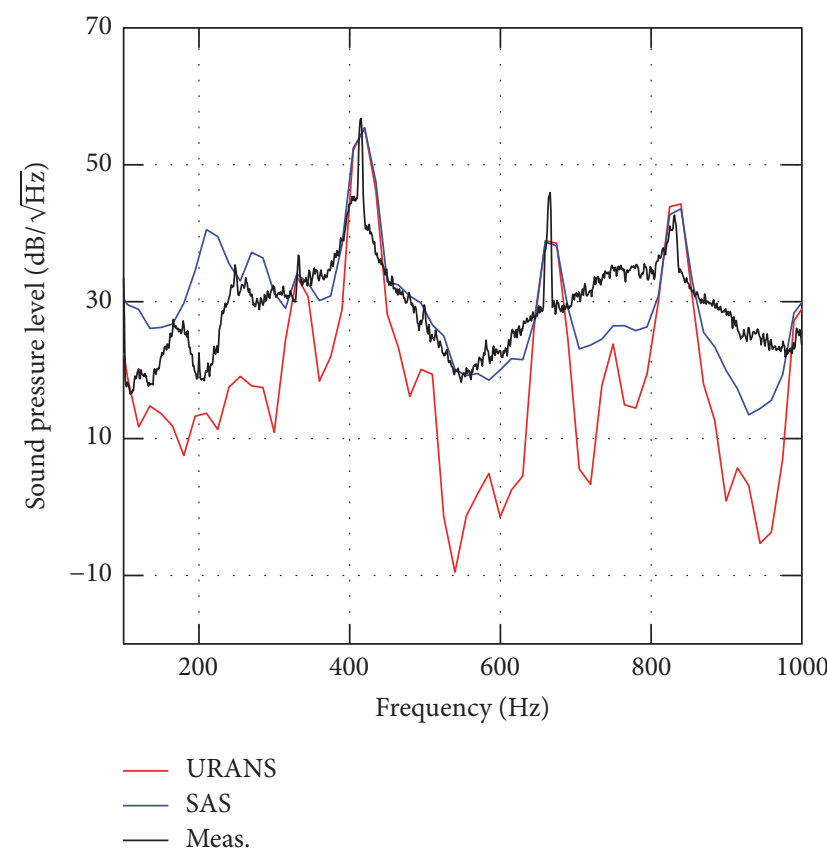

(a) Inflow region

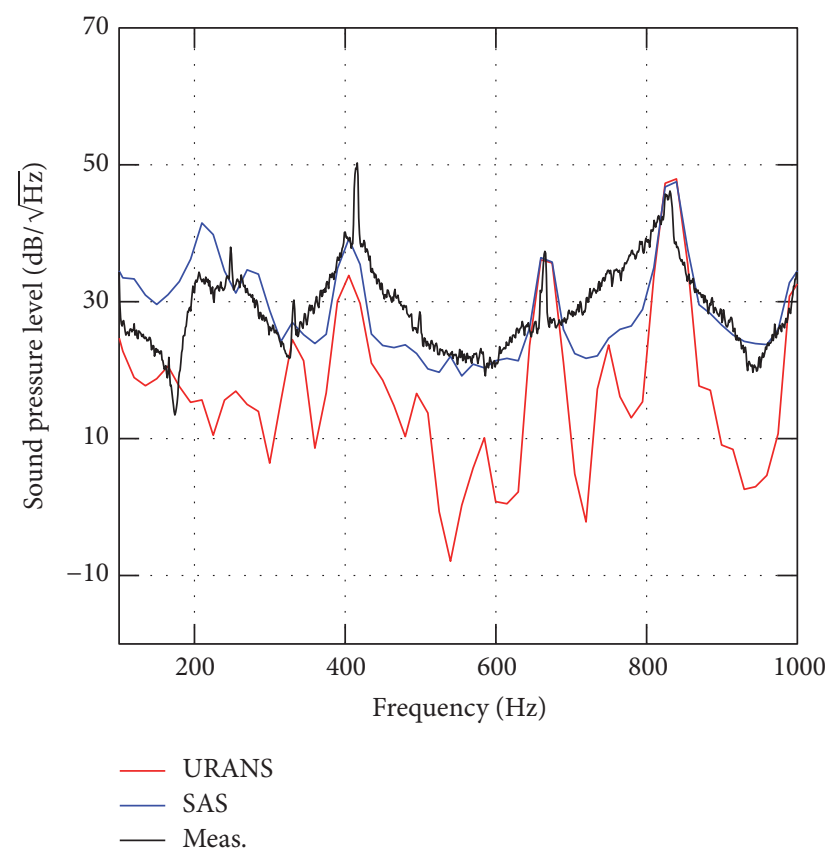

(b) Outflow region

FIGURE 23: Comparison of acoustic spectra: sound pressure levels: inflow: $\mathrm{SPL}_{\text {meas. }}=66.0 \mathrm{~dB} ; \mathrm{SPL}_{\mathrm{URANS}}=69.8 \mathrm{~dB} ; \mathrm{SPL}_{\mathrm{SAS}}=70.2 \mathrm{~dB} ;$ outflow: $\mathrm{SPL}_{\text {meas. }}=63.7 \mathrm{~dB} ; \mathrm{SPL}_{\text {URANS }}=63.1 \mathrm{~dB} ; \mathrm{SPL}_{\mathrm{SAS}}=64.7 \mathrm{~dB}$.

TABLE 1: Explanation of peaks in the acoustic spectra.

\begin{tabular}{|c|c|c|}
\hline $\begin{array}{l}\text { Frequency } \\
\text { in } \mathrm{Hz}\end{array}$ & Trigger & Annotation \\
\hline 215 & Acoustic cavity eigenmode & $\begin{array}{l}\text { Front panel slot; } \\
\text { Figure 24(a) }\end{array}$ \\
\hline 278,403 & $\begin{array}{l}\text { Acoustic cavity } \\
\text { eigenmodes }\end{array}$ & $\begin{array}{l}\text { Card slots; Figures } \\
24(\mathrm{~b}) \text { and } 24(\mathrm{c})\end{array}$ \\
\hline 416 & Acoustic cavity eigenmode & $\begin{array}{l}\text { Mainboard slot; } \\
\text { Figure } 24(\mathrm{~d})\end{array}$ \\
\hline 417,833 & Blade passing frequency & $\begin{array}{c}\text { Including higher } \\
\text { harmonics; Figure } 25\end{array}$ \\
\hline $\begin{array}{l}333,667 \\
1000,1333\end{array}$ & Rotor-stator interaction & $\begin{array}{c}\text { Including higher } \\
\text { harmonics; Figure } 27\end{array}$ \\
\hline 1666 & Blade-stator interaction & - \\
\hline 2330 & First structural eigenmode & At $2320 \mathrm{~Hz}$; Figure 9(a) \\
\hline 2490 & $\begin{array}{c}\text { Second structural } \\
\text { eigenmode }\end{array}$ & At $2484 \mathrm{~Hz}$; Figure 9(b) \\
\hline
\end{tabular}

first and second structural eigenmodes with frequencies of 2320 and $2484 \mathrm{~Hz}$, respectively (see Figure 9). These two effects can only be identified in the spectra of the measured sound pressure (Figure 22) having a higher frequency limit. A summary of the effects resulting in a tonal sound emission for simulation and measurement is given in Table 1.

Comparing the acoustic spectra of URANS-SST and SASSST, again the consequences of the different modeling techniques become obvious: While the URANS-SST simulation shows higher damping of less dominant turbulent structures (cf. Figures 11, 12, 13, 14, 16, 19, and 20), part of these structures is resolved with the SAS-SST model, also resulting in a better estimation of the acoustics at those frequencies. It is evident that the scale resolving SAS case can account far better for the broadband noise in between the dominant effects like blade passing and rotor-stator interaction. The overall sound pressure levels for the frequency range between 100 and $1000 \mathrm{~Hz}$ are given in Figure 23. Both simulations tend to overestimate the sound pressure level at the inlet. The overall sound pressure levels show that results based on the URANS-SST simulation are closer to the measured values than those based on the SAS-SST simulation. The reason is a compensation of errors of the sound pressure at the blade passing frequency $(56.8 \mathrm{~dB})$ and its first harmonic ( $42.6 \mathrm{~dB}$ ) with the SAS-SST model ( $55.3 \mathrm{~dB}$ and $43.6 \mathrm{~dB}$, resp.) compared with URANS-SST model $(55.4 \mathrm{~dB}$ and $44.3 \mathrm{~dB}$, resp.). Both simulations underestimate the sound pressure level at the blade passing frequency and overestimate the sound pressure level at the first harmonic of the blade passing frequency. At the outlet the overall sound pressure level is an error range of $\pm 1 \mathrm{~dB}$ while at the inlet the error is within $\pm 4.2 \mathrm{~dB}$. The sound pressure simulated for the rotor-stator interaction and its higher harmonics is in general too low with the SAS simulation being in slightly better agreement than the URANS case.

\section{Conclusions}

CFD and CAA simulations of the air-cooling of a generic electronic device were performed. The flow field was simulated using ANSYS CFX and the sound propagation was calculated using the in-house FE solver CFS++. The acoustic 


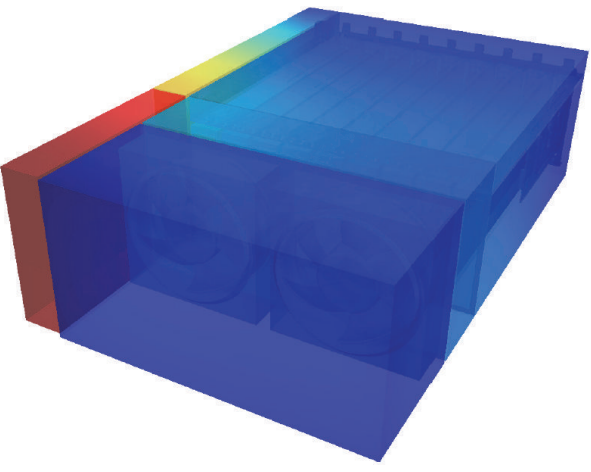

(a) $215 \mathrm{~Hz}$

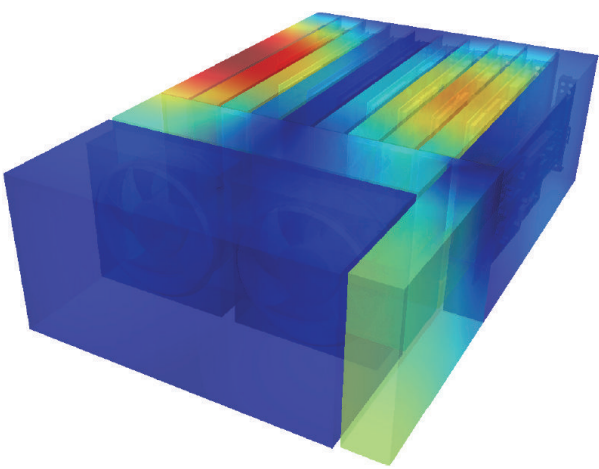

(c) $403 \mathrm{~Hz}$

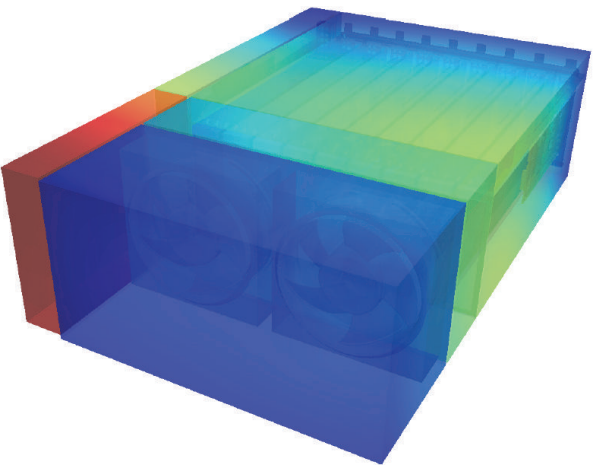

(b) $278 \mathrm{~Hz}$

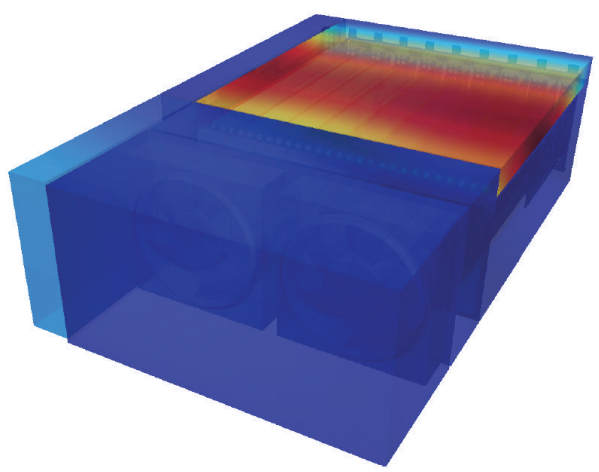

(d) $416 \mathrm{~Hz}$

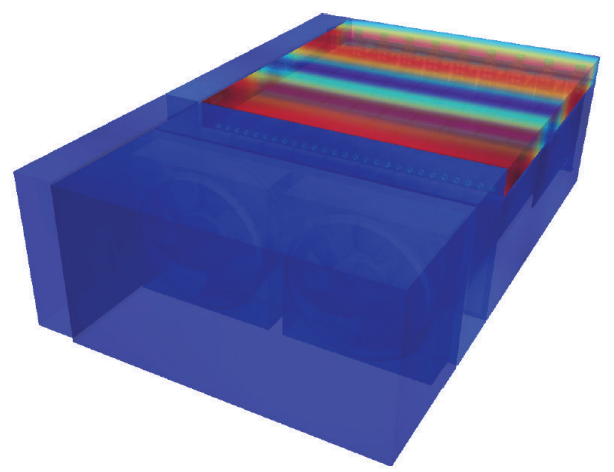

(e) $868 \mathrm{~Hz}$

FIGURE 24: Acoustic eigenmodes determined via modal analysis of normalized sound pressure (blue: $0 ;$ red: 1 ).

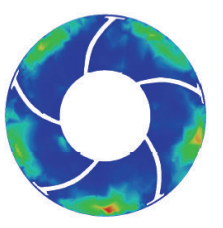

(a) Acoustic sources
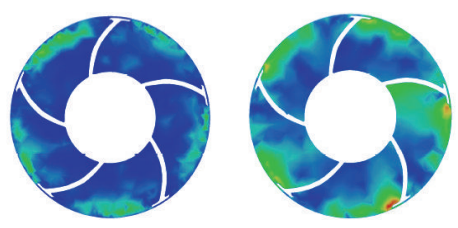

(b) Sound pressure

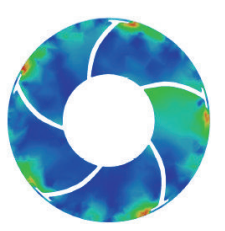

pressure

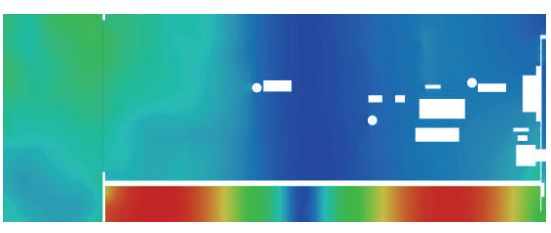

(c) Sound pressure

FIGURE 25: Normalized discrete Fourier transform of the aeroacoustic sources computed according to the right-hand side of (4) (a) and sound pressure field computed by the CAA method based on URANS data at $837 \mathrm{~Hz}$ on plane perpendicular to the fan axes (b) and on a vertical midplane through the device (c) (blue: 0; red: 1 ). 


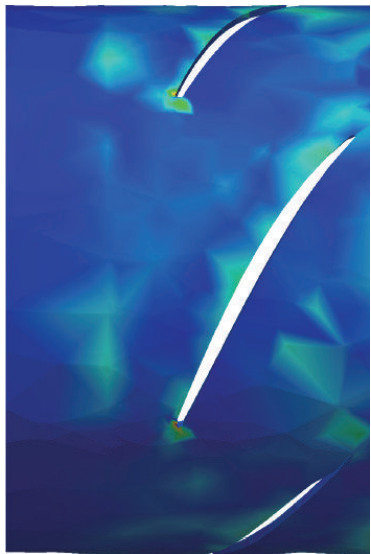

(a) Acoustic sources at $95 \mathrm{~Hz}$

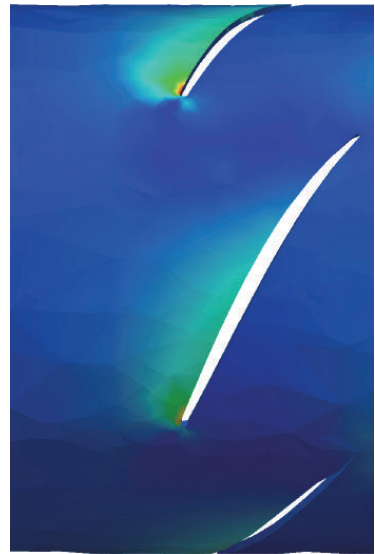

(b) Sound pressure at $95 \mathrm{~Hz}$

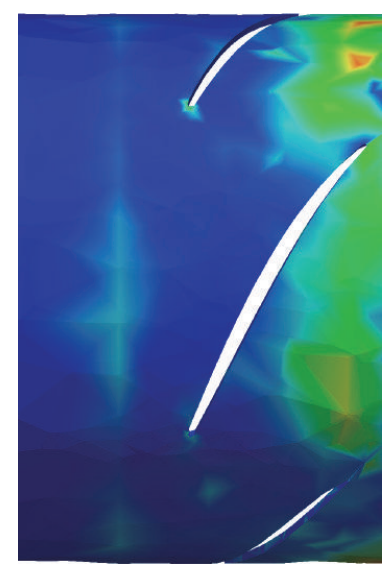

(c) Acoustic sources at $247 \mathrm{~Hz}$

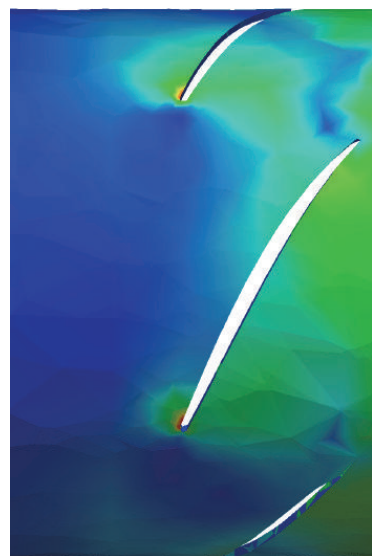

(d) Sound pressure at $247 \mathrm{~Hz}$

FIGURE 26: Normalized discrete Fourier transform of the aeroacoustic sources computed according to the right-hand side of (4) and sound pressure field computed by the CAA method based on URANS data at $95 \mathrm{~Hz}(\mathrm{a}, \mathrm{b})$ and $247 \mathrm{~Hz}(\mathrm{c}, \mathrm{d})$ on a cylindrical plane concentric to the fan axis at a radius of $35 \mathrm{~mm}$ (blue: 0 ; red: 1 ).

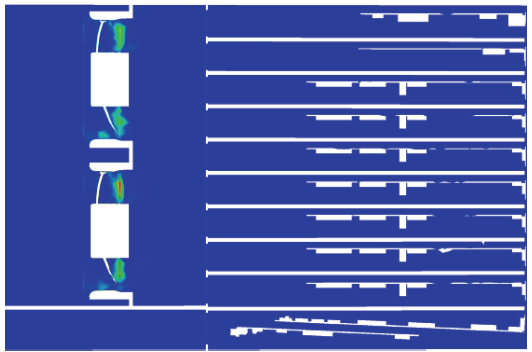

(a) Acoustic sources

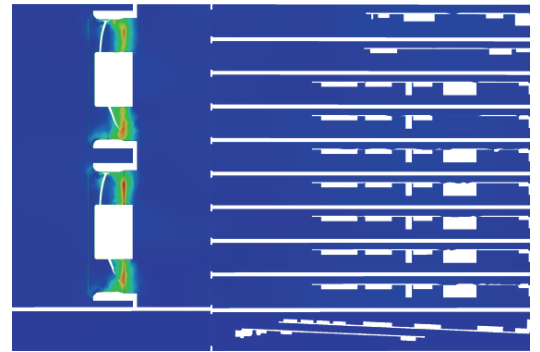

(b) Sound pressure

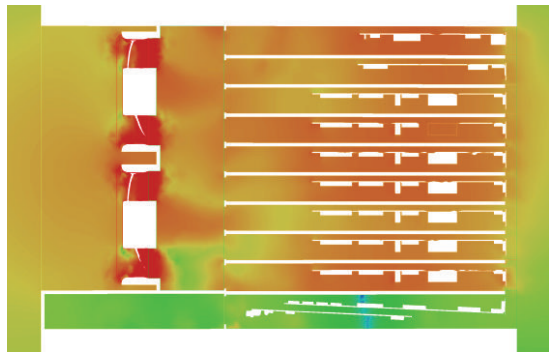

(c) Sound pressure normalized to $\max / 4$ with logarithmic scale

FIGURE 27: Normalized discrete Fourier transform of the aeroacoustic sources computed according to the right-hand side of (4) and sound pressure field computed by the CAA method based on URANS data at $666 \mathrm{~Hz}$ on plane through both fan axes (blue: 0; red: 1).

results were compared with measurements and showed good agreement. In the case of the CFD simulation, two different turbulent modeling approaches were used: URANS-SST and SAS-SST. Both CFD and CAA simulations demonstrated that the SAS approach is capable of resolving turbulent scales in a more detailed fashion, provided that the spatial discretization (CFD element size) and temporal resolution (time stepping) have been chosen properly. The acoustic source terms were calculated according to Lighthill's acoustic analogy.

The acoustic results with the SAS-SST approach are in better agreement with measurements than the CAA results based on the URANS-SST approach, even though the overall sound pressure level of the URANS-SST is in better agreement with the measurement as the errors compensate each other in a positive manner. The estimated overall sound pressure levels show a maximum error of only $4.2 \mathrm{~dB}$ whereas the best estimation is merely $0.6 \mathrm{~dB}$ too low. The acoustic peaks in the spectra were linked to different effects, that is, blade passing frequency, rotor-stator interaction, blade-stator interaction, and structural and acoustic eigenmodes of the system.
Even though the results are in good agreement with the measurements, especially the reproduction of the spectra in the SAS case, a more precise estimation might be achieved by accounting for three aspects. First, as already mentioned in the CFL section of this paper, the time step should be further decreased to achieve a CFL number below 1 in the whole simulation domain, especially in the fan domains where most of the acoustic sources are generated. This will additionally account for a better temporal resolution of the acoustic simulation and hence allow resolving up to higher frequencies. Secondly, the acoustic domain should be modified to meet the same conditions as the experiments, that is, an acoustic domain that enables acoustic transmission from inlet to outlet outside of the electronic device instead of the modeled rigid wall. Thirdly, a perfectly matching layer (PML) should be introduced to avoid reflections. This will produce more accurate results especially in the frequency range below approx. $400 \mathrm{~Hz}$.

\section{Competing Interests}

The authors declare that they have no competing interests. 


\section{Acknowledgments}

This work was supported by the Bayerische Forschungsstiftung (BFS, Bavarian Research Foundation) within the project "FORLärm-Lärmminderung von technischen Anlagen" (Noise Reduction in Technical Equipment) under Grant no. 890-09.

\section{References}

[1] M. Kaltenbacher, Numerical Simulation of Mechatronic Sensors and Actuators: Finite Elements for Computational Multiphysics, Springer, Berlin, Germany, 2015.

[2] E. Baugh, "Acoustic limitations in notebook thermal design," in Proceedings of the 10th Electronics Packaging Technology Conference (EPTC '08), pp. 725-730, IEEE, Singapore, December 2008.

[3] M. Nantais, C. Novak, and J. Defoe, "Graphics processing unit cooling solutions: acoustic characteristics," Canadian Acoustics, vol. 34, no. 3, pp. 78-79, 2006.

[4] L. Huang, "Characterizing computer cooling fan noise," The Journal of the Acoustical Society of America, vol. 114, no. 6, pp. 3189-3200, 2003.

[5] L. Huang and J. Wang, "Acoustic analysis of a computer cooling fan," Journal of the Acoustical Society of America, vol. 118, no. 4, pp. 2190-2200, 2005.

[6] J. Defoe and C. Novak, "Review of computational aeroacoustics for application in electronics cooler noise," Canadian Acoustics, vol. 34, no. 3, pp. 76-77, 2006.

[7] F. R. Menter, "Two-equation eddy-viscosity turbulence models for engineering applications," AIAA Journal, vol. 32, no. 8, pp. 1598-1605, 1994.

[8] B. E. Launder and D. B. Spalding, Mathematical Models of Turbulence, Academic Press, London, UK, 1972.

[9] A. Kolmogorov, "Equations of turbulent motion of an incompressible fluid," Izvestiya Akademii Nauk SSSR, Seriya Fizicheskaya, vol. 6, pp. 56-58, 1942.

[10] J. C. Rotta, Turbulente Strömungen, Teubner, Stuttgart, Germany, 1972.

[11] F. Menter and Y. Egorov, "Revisiting the turbulent scale equation," in Proceedings of the IUTAM Symposium on One Hundred Years of Boundary Layer Research, pp. 279-290, 2004.

[12] F. Menter and Y. Egorov, "A scale adaptive simulation model using two-equation models," in Proceedings of the 43rd AIAA Aerospace Sciences Meeting and Exhibit, AIAA paper 2005-1095, Reno, Nev, USA, January 2005.

[13] Y. Egorov, "Menter, development and application of SST-SAS turbulence model in the DESIDER project," in Proceedings of the 2nd Symposium on Hybrid RANS-LES Methods, Corfu, Greece, 2007.

[14] F. R. Menter and Y. Egorov, “The scale-adaptive simulation method for unsteady turbulent flow predictions. Part 1: theory and model description," Flow, Turbulence and Combustion, vol. 85, no. 1, pp. 113-138, 2010.

[15] M. J. Lighthill, "On sound generated aerodynamically. I. General theory," Proceedings of the Royal Society of London, Series A: Mathematical and Physical Sciences, vol. 211, no. 1107, pp. 564587, 1952.

[16] M. Kaltenbacher, M. Escobar, S. Becker, and I. Ali, "Numerical simulation of flow-induced noise using LES/SAS and Lighthill's acoustic analogy," International Journal for Numerical Methods in Fluids, vol. 63, no. 9, pp. 1103-1122, 2010.
[17] S. Triebenbacher, M. Kaltenbacher, M. Escobar, and B. Flemisch, "Nonmatching grids for the coupled computation of flow induced noise," in Proceedings of the 13th AIAA/CEAS Aeroacoustics Conference, AIAA 2007-3512, Rome, Italy, May 2007. 


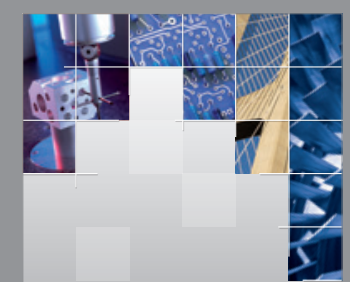

\section{Enfincering}
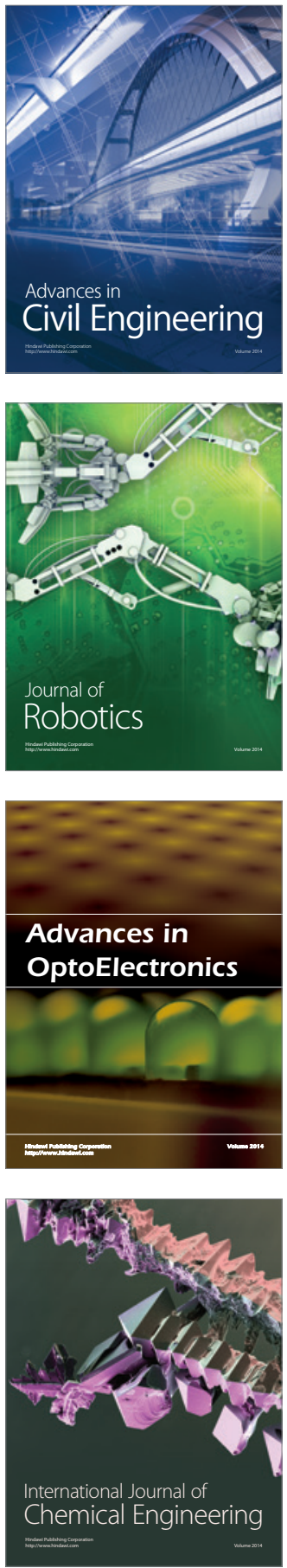

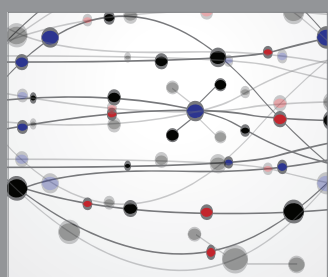

The Scientific World Journal

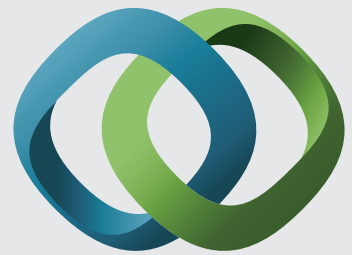

\section{Hindawi}

Submit your manuscripts at

http://www.hindawi.com
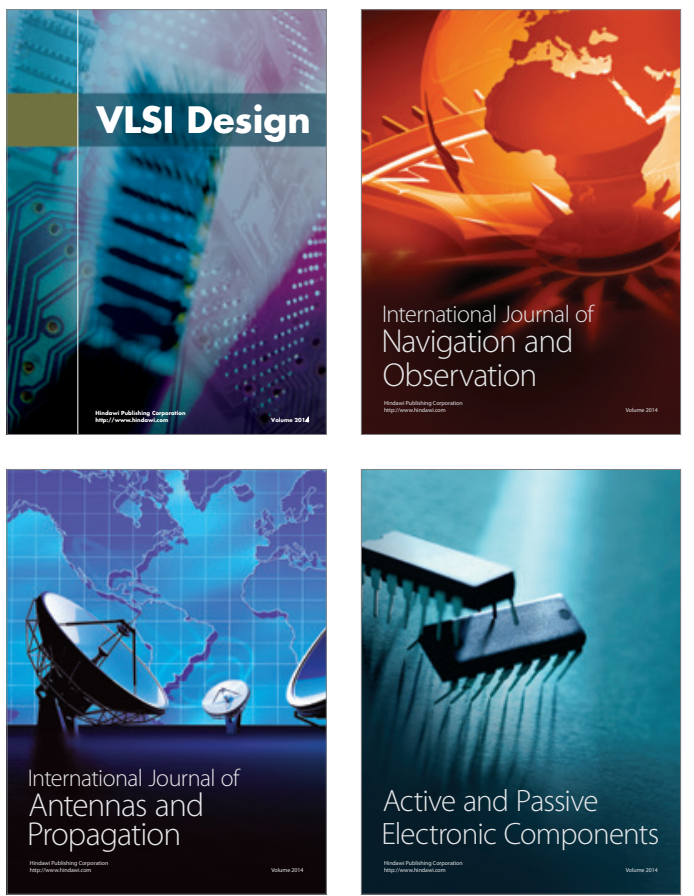
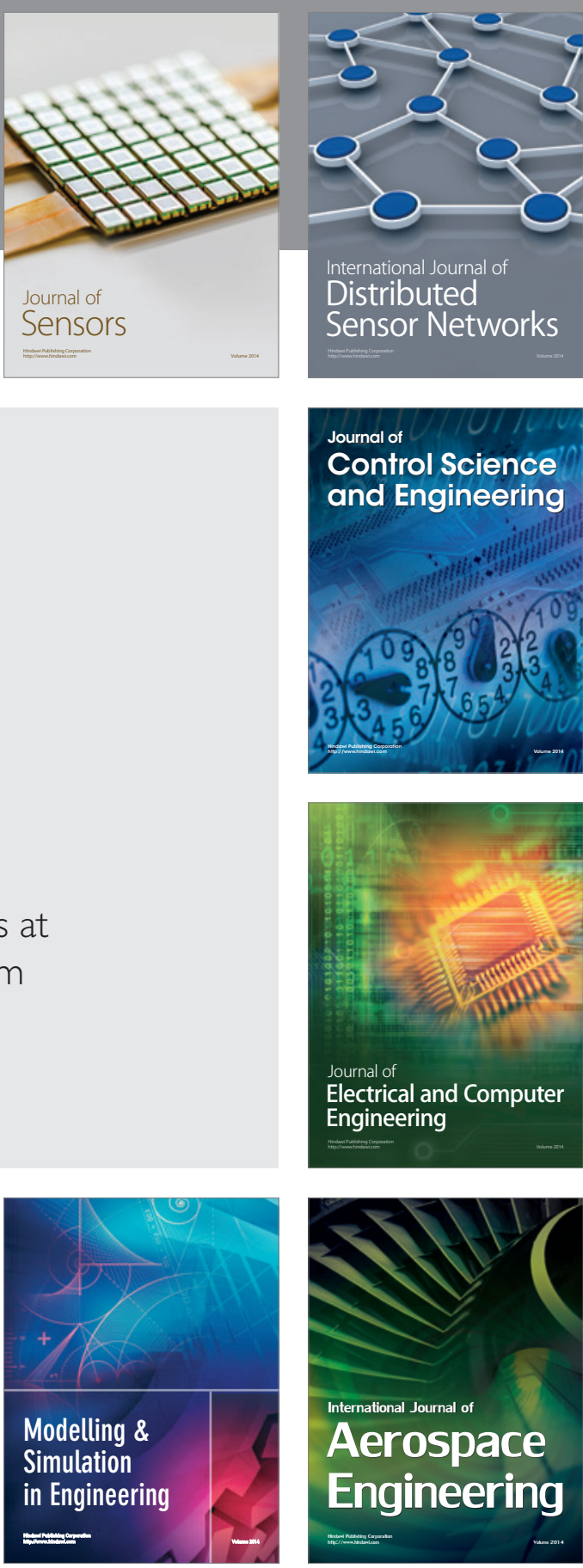

International Journal of

Distributed

Sensor Networks

Journal of

Control Science

and Engineering
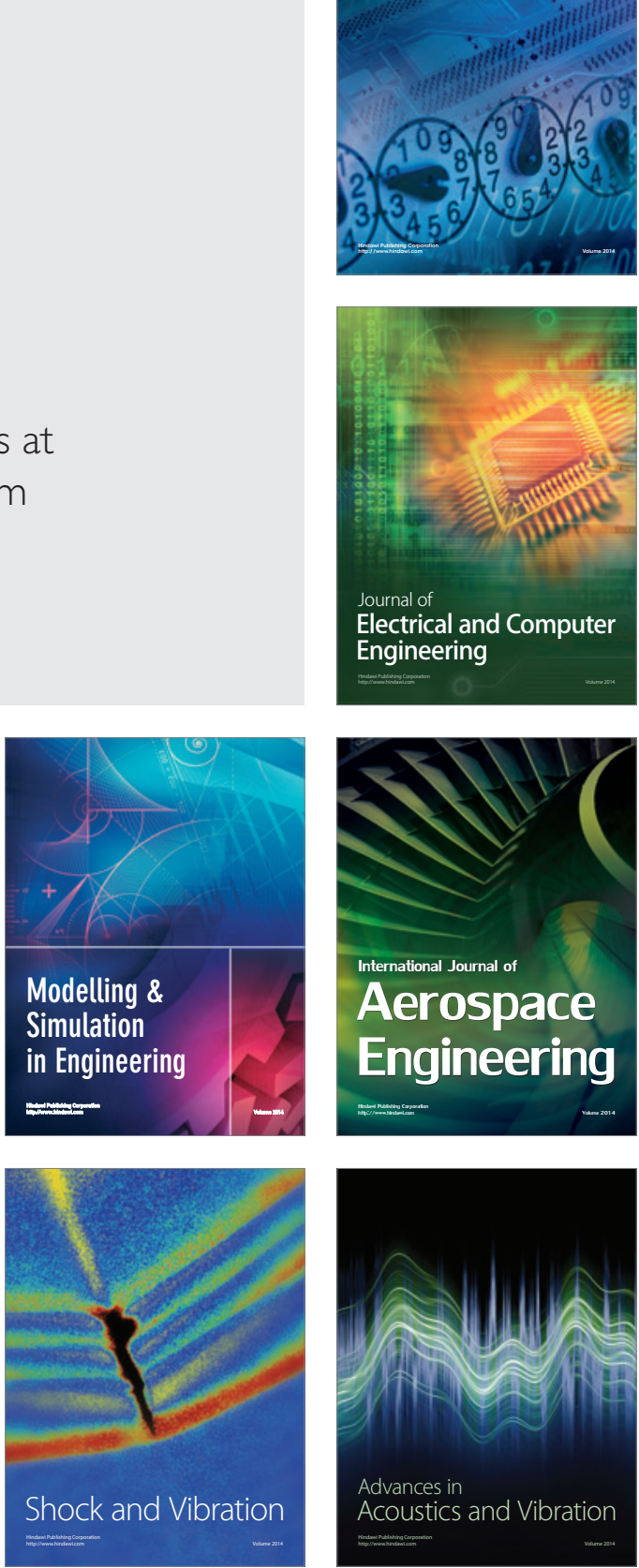